\title{
Das Unsichtbare begreifen
}

\author{
Die Rekonstruktion historischer Wahrnehmungsmodi \\ als methodische Herausforderung der Kulturgeschichte*
}

Von

Silvia Serena Tschopp

I.

Was die Kulturgeschichte zu leisten habe, so Rudolf Vierhaus in einem vor einigen Jahren veröffentlichten Aufsatz über ,Probleme moderner Kulturgeschichtsschreibung', sei „die möglichst vollständige Rekonstruktion der Bedingungen, Anlässe, Formen, Ergebnisse und Folgen sinnhaften Handelns konkreter Menschen - Individuen und Gruppen in der Vergangenheit." Dabei habe sie den Fokus nicht nur auf jene Gegenstandsbereiche zu richten, welche die politische Ereignisgeschichte und die Sozialgeschichte als Forschungsfeld beanspruchten, sondern auch und vor allem auf ,die Formen der Wahrnehmung von Wirklichkeit, ihrer Deutung und Gestaltung durch Wissen; $[\ldots \mid$ die Denkformen und Handlungsspielräume der Menschen unter den jeweiligen konkreten geschichtlichen Bedingungen, die psychisch und kulturell konstituierten Formen und verhaltenssteuernden Wirkungen der Empfindungen, der emotionalen Sensibilitäten, des Glaubens, des Bewußtseins der Menschen." 2 Darüber, daß die Aufgabe der Kulturgeschichte nicht primär in der Erforschung konkreter historischer Begebenheiten und - um mit Hegel zu sprechen - welthistorischer Indivi-

\footnotetext{
"Erweiterte Fassung meiner Antrittsvorlesung als Professorin für Europäische Kulturgeschichte an der Universität Augsburg. Dank gebührt jenen Kolleginnen und Kollegen, allen voran Achim Landwehr, die mir als kritische Gesprächspartner vielfältige Anregungen vermittelt haben.

1 Rudolf Vierhaus, Dic Rekonstruktion historischer Lebenswelten. Probleme moderner Kulturgeschichtsschreibung, in: Wege zu einer neuen Kulturgeschichte. Mit Beitr. v. Rudolf Vierhaus u. Roger Charticr. (Göttinger Gespräche zur Geschichtswissenschaft, 1.) Göttingen 1995, 7-28, hier 16.
}

2 Fbd. $8 \mathrm{f}$. 
duen ${ }^{6} 3$ besteht, herrscht unter Historikern weitgehender Konsens. Seit Voltaire in seinem „Essai sur l'histoire générale et sur les mœurs et l'ésprit des nations" (1756) erstmals das Programm einer Geschichtsschreibung formulierte, die sich nicht auf Schlachten, auf Könige und Generäle oder auf Hofgeschichten zu reduzieren, sondern die in einem viel umfassenderen Sinn menschliche Erfahrung und die sie bedingenden und strukturierenden mentalen Konstellationen darzustellen habe, postuliert die Kulturgeschichte ihre Zuständigkeit für jene Vorstellungen, Werthaltungen, Wissensbestände und Wahrnehmungsmuster, welche im historischen Handeln von Individuen und Kollektiven zum Ausdruck gelangen. Giambattista Vico, Johann Gottfried Herder, Johann Christoph Adelung oder im 19. Jahrhundert Jacob Burckhardt, jene Schriftsteller, Philosophen und Wissenschaftler also, denen als Repräsentanten der, älteren' Kulturgeschichte mittlerweile ein kanonischer Status zukommt, konvergieren ungeachtet der Heterogenität ihrer Positionen in der Emphase, mit der sie die geistige Natur des Menschen als Triebkraft geschichtlichen Handelns postulieren und die daraus resultierenden kulturellen Praktiken in den Mittelpunkt ihres Erkenntnisinteresses rücken. Und auch jene Vertreter der Kultursoziologie und Kulturphilosophie, die sich im frühen 20. Jahrhundert um eine Dynamisierung der kulturhistorischen Reflexion bemühen und an der Herausbildung einer , neuen Kulturgeschichte' in erheblichem Maße partizipieren - zu nennen wären beispielsweise Max Weber, Georg Simmel oder Ernst Cassirer - definieren Geschichte als einen Geschehenszusammenhang, der sich wesentlich ideellen Stimuli verdankt.

Indem sie historische Ereignisse und Prozesse in einen ursächlichen Zusammenhang mit geschichtlich mehr oder weniger spezifischen individuellen und kollektiven Vorstellungswelten stellen, handeln sich Kulturhistoriker allerdings ein nicht zu unterschätzendes methodisches Problem ein: Inwicfern lassen sich immaterielle Phänomene wie menschliches Denken und Fühlen erfassen und auf eine Art und Weise darstellen, die Anspruch auf wissenschaftliche Relevanz erheben darf? Wie kann etwas derart Geschichtsmächtiges und zugleich Ungreitbares wie menschliche Wahrnehmung als wissenskonstituierende und handlungsleitende Operation auf wissenschaftlich plausible Weise rekonstruiert werden? Welcher Instrumente soll sich der Geschichtsforscher

${ }^{3} \mathrm{Vgl}$. Georg Friedrich Wilhelm Hegel, Vorlesungen über die Philosophic der Geschichte. (Werke. Bd. 12.) Frankfurt am Main 1970, 45. 
bedienen, wenn er versucht, den geistigen Triebkräften historischen Handelns auf die Spur zu kommen? Eine Antwort auf die hier gestellten Fragen hatte bereits Wilhelm von Humboldt versucht. In seinem Aufsatz „Über die Aufgabe des Geschichtschreibers“ betont er, daß das „Geschehene $[\ldots]$ nur zum Theil in der Sinnenwelt sichtbar" sei. ${ }^{4}$ ,,[D]as Uebrige“ müsse „,hinzu empfunden, geschlossen, errathen werden"5, denn ,[d]ie Wahrheit alles Geschehenen beruht auf dem Hinzukommen jenes oben erwähnten Theils jeder Thatsache, und diesen muß daher der Geschichtschreiber hinzufügen" 6 . Was Wilhelm von Humboldt vom Historiographen fordert, sind folgerichtig „Ahndungsvermögen" und „Verknüpfungsgabe" ${ }^{\text {"7 }}$ als jene Erkenntnisinstrumente, deren es bedürfe, um eine sich in fragmentarischer Form präsentierende ,Wirklichkeit ' adäquat zu erfassen. Zwar betont auch Humboldt die Notwendigkeit einer ,genauen, partheilosen, kritischen Ergründung des Geschehenen“, zur Wahrheit könne der Historiker jedoch nur vordringen, wenn er diese Operation verbinde mit dem „Ahnden des durch jene Mittel nicht Errcichbaren“..

Eine derartige aus Analyse und Imagination hervorgehende Historiographie stieß3 im Zuge der Institutionalisierung der Geschichtswissenschaft als akademischer Disziplin bei Historikern auf zunehmenden Widerspruch. Der mit einer Hinwendung zu positivistischen Positionen einhergehende Siegeszug der quellenkritischen Forschung zementierte im 19. Jahrhundert ein Wissenschaftsideal, das methodische Rationalität als konstitutiv für forschendes Bemühen postuliert. Historische Erkenntnis hat sich nun auf einen realen, aus den Quellen ermittelbaren Zusammenhang, den der Forscher im Zuge methodisch bewußter Operationen sichtbar macht, zu stützen. Für Leopold von Ranke, mit dessen Namen sich der für den deutschen Historismus kennzeichnende epistemologische Wandel innerhalb der Geschichtswissenschaft vor allem verbindet. liegt die Aufgabe des Historikers darin, unter größtmöglicher Ausschaltung seiner Subjektivität zu ,zeigen, wie es eigentlich ge-

+ Wilhelm von Humboldt, Ueber die Aufgabe des Geschichtschreibers, in: Wilhelm von Humboldts gesammelte Schriften. Hrsg. v. der Königlich Preussischen Akademic der Wissenschaften. Bd. 4. Berlin 1905, 35-36, hier 35.

5 Fibd.

6 Ebd. 36 .

7 Fhd. 37.

${ }^{8}$ Ebd. 
wesen". ${ }^{9}$ Zwar war sich Ranke der Standortgebundenheit geschichtlicher Forschung in weit höherem Maße bewußt, als dies die spätere Ranke-Forschung vermuten ließ $\mathrm{e}^{10}$, und das Vertrauen in die Fähigkeit des Historiographen, durch systematische Erschließung der Quellen und deren kritische Analyse eine objektive Darstellung vergangenen Geschehens zu liefern, wird bereits von dessen Zeitgenossen problematisiert ${ }^{11}$. Die Forderung nach einer Privilegierung der Primärquellen,

${ }^{9}$ Leopold von Ranke, Geschichten der romanischen und germanischen Völker 1494-1514, in: Leopold von Ranke's Sämmtliche Werke. Hrsg. v. Alfred Dove. Bd. 33. 4. Aufl. Leipzig 1874, VII [Vorrede zur ersten Ausgabe, Oktober 1824].

10 Vgl. dazu beispielsweise Daniel Fulda, Wissenschaft aus Kunst. Die Entstehung der modernen deutschen Geschichtsschreibung 1760-1860. (European Cultures, 7.) Berlin/New York 1996, 296-343, und Johannes Süßmann, Geschichtsschreibung oder Roman? Zur Konstitutionslogik von Geschichtserzählungen zwischen Schiller und Ranke (1780-1824). (Frankfurter Historische Abhandlungen, 41.) Stuttgart 2000, 215-256.

11 Mit der Theorie vom Sehepunckt hat bereits Johann Martin Chladenius, Allgemeine Geschichtswissenschaft. Leipzig 1752, die unausweichliche Standortgebundenheit historischer Betrachtung in den Blick gerückt. Vgl. dazu Reinhart Kosel$l e c k$, Standortbindung und Zeitlichkeit. Ein Beitrag zur historiographischen Erschließung der geschichtlichen Welt, in: ders./Wolfgang J. Mommsen/Jörn Rüsen (Hrsg.), Objektivität und Parteilichkeit in der Geschichtswissenschaft. (Theorie der Geschichte. Beiträge zur Historik, 1.) München 1977, 17-46; Christoph Friedrich, Sprache und Geschichte. Untersuchungen zur Hermeneutik von Johann Martin Chladenius. (Studien zur Wissenschaftstheorie, 13.) Meisenheim am Glan 1978; sowie neuerdings Eckhard Höfner, J. M. Chladenii „Sehepunckt". Zu Problemen der historischen Erklärung in der Allgemeinen Geschichtswissenschaft und in heutiger Interpretation, in: Alfonso de Toro/Stefan Welz (Hrsg.), Rhetorische Seh-Reisen. Fallstudien $z u$ Wahrnehmungsformen in Literatur, Kunst und Kultur. (Leipziger Schriften zur Kultur-, Literatur-, Sprach- und Übersetzungswissenschaft, 9.) Frankfurt am Main 1999, 9-40. Auch für dic historistische Geschichtswissenschaft erweist sich die Subjektivität menschlicher Wahrnehmung und Deutung als fundamentales Problem historiographischer Tätigkeit. So verweist Johann Gustav Droysen in seiner Historik (postum 1882) auf die Tatsache, ,daß uns die Vergangenheiten nicht mehr unmittelbar, sondern nur in vermittelter Weise vorliegen, daß wir nicht, objektiv' die Vergangenheiten, sondern nur aus den, Quellen' eine Auffassung, eine Anschauung, ein Gegenbild von ihnen herstellen können, daß die so gewinnbaren und gewonnenen Auffassungen und Anschauungen alles sind, was uns von der Vergangenheit zu wissen möglich ist, daßß also, dic Geschichte ' nicht äußßerlich und realistisch. sondern nur so vermittelt, so erforscht und so gewulst da ist", und betont in diesem Zusammenhang die Notwendigkeit, das ,unmittelbare und subjektive Auffassen“ des Geschichtsforschers durch ,objektive Maße und Kontrollen" zu regulieren. Vgl. Johamn Gustav Droysen, Historik. Historisch-kritische Ausgabe. Rekonstruktion der ersten vollständigen Fassung der Vorlesungen (1857). Grundrib der Historik in der ersten handschriftlichen (1857/1858) und in 
nach einem kritischen Umgang mit historischen Dokumenten, nach einer Darstellungsweise, welche akademischen Maßstäben genügt und durch argumentative Transparenz den intellektuellen Nachvollzug der gewonnenen Erkenntnisse ermöglicht, offenbart allerdings einen bemerkenswerten Optimismus hinsichtlich der Möglichkeiten historischer Erkenntnis. ,Objektivität' wird zu einem Leitbegriff geschichtlicher Forschung, was nicht nur die Gewinnung neuer Methoden für die Geltungssicherung des jeweils Erforschten begünstigt, sondern auch zu einer Neubestimmung des Gegenstandbereichs der Geschichtswissenschaft führt. Im Zentrum historiographischen Bemühens stehen nun immer ausschließlicher jene Ereignisse und Persönlichkeiten, welche durch die in Archiven sowie gedruckten Quellensammlungen zugänglich gemachten Dokumente bezeugt sind. Was mit Hilfe herkömmlicher geschichtlicher Überlieferung und des methodischen Instrumentariums der Quellenkritik nicht erhellt werden kann, gerät aus dem Blickfeld des Historikers. In cinem Moment, in dem die Geisteswissenschaften gegenüber den Naturwissenschaften in zunehmendem Maße in die Defensive geraten und ihr methodisches Fundament verstärktem Legitimationsdruck ausgesetzt sehen, scheint es außerdem opportun, sich auf jene Fragestellungen zu konzentrieren, die eine der Forderung nach Wissenschaftlichkeit genügende Antwort erlauben. ${ }^{12}$ Damit rückt in den Hintergrund, was seit ihren Anfängen das genuine Feld der Kulturgeschichte ausmachte. Wer sich im 19. Jahrhundert unter kulturhistorischer Perspektive mit der Vergangenheit und den darin sich manifestierenden mentalen Dispositionen befaßt, darf zwar in der Regel mit Erfolg bei cinem breiteren Publikum rechnen, von akademischen Kollegen jedoch wird ihm vorgeworfen, mit seinem Vorgehen den szientifischen Charakter historischer Forschung zu untergraben. Die kontrovers geführten Auseinandersetzungen zu Methodenproblemen geschichtlicher Forschung im 19. und auch im 20. Jahrhundert führen in

der letzten gedruckten Fassung (1882). Hrsg. v. Peter Leyh. Bd. I. Stutgart-Bad Cannstatt 1977, 484 u. 486.

12 Zum belasteten Verhältnis zwischen den zunehmend in den Rang von Leitwissenschaften erhobenen naturwissenschaftlichen Disziplinen und der Geschichtswissenschaft im 19. Jahrhundert und den sich daraus ergebenden methodologischen Konsequenzen für die historische Forschung vgl. Otoo Gerhard Oexle. Naturwissenschaft und Geschichtswissenschaft. Momente einer Problemgeschichte. in: ders. (Hrsg.). Naturwissenschaft. Geisteswissenschaft, Kulturwissenschaft. Einheit - Gegensal\% .. Komplementarität) (Göttinger Gespräche zur Geschichtswissenschaft. 6.) Göttingen 1998. 99-151. 
Deutschland zunächst eher zu einer Verfestigung als zu einer Relativierung des seit den Anfängen des Historismus den theoretischen Diskurs dominierenden Wissenschaftsideals. ${ }^{13}$ Die Frage nach der Ermittelbarkeit und Darstellbarkeit jenes Bereichs von ,Wirklichkeit ", welcher für den Historiker unsichtbar bleibt, die Wilhelm von Humboldt noch in den Mittelpunkt seiner wissenschaftstheoretischen Erörterungen gestellt hatte, findet in der Folge nicht nur keine überzeugende Antwort, sie erscheint nicht wenigen Historikern geradezu als suspekt oder zumindest als irrelevant.

Der ,kulturalistischen Wende", die mittlerweile auch in Deutschland die Geisteswissenschaften erfaßt hat, ist es zu verdanken, daß das hier interessierende Problem an Signifikanz gewann und innerhalb der Forschungsdiskussion erneut thematisiert wird. Nachdem ,Mentalitäten', religiöse, politische, ökonomische und soziale Vorstellungen und Handlungsnormen insbesondere in Frankreich und Italien sowie im angelsächsischen Raum zu einem bevorzugten Thema geschichtlicher Forschung avancierten, erwies sich die Klärung der Frage, wie sich menschliches Denken und Empfinden methodisch überzeugend begreifen und darstellen lasse, als vordringliche Aufgabe kulturhistorischer Reflexion. Von einer wirklich befriedigenden Antwort sind wir, wie mir scheint, noch weit entfernt, hingegen ist es zum jetzigen Zeitpunkt möglich, die Schwierigkeiten, die sich demjenigen in den Weg stellen, der eine wissenschaftlich fundierte Erfassung und Beschreibung historischer Denkformen und Deutungsmuster anstrebt, präziser zu benennen und dic Richtung anzudeuten, in welche methodologische Bemühungen in Zukunft gehen könnten. An diesem Punkt setze ich mit meinen Ausführungen an: Ausgehend von Überlegungen des französischen Historikers Roger Chartier werde ich in einem ersten Schritt darlegen. worin die Schwierigkeiten einer historischen Rekonstruktion menschlicher Vorstellungswelten liegen, bevor ich in einem zweiten Schritt

13 7um ,Methodenstreit" innerhalb der Geschichtswissenschaft um die Wende vom 19. zum 20. Jahrhundert vgl. vor allem Stefan Hats. Historische Kulturforschung in Deutschland 1880-1930. Geschichtswissenschaft zwischen Synthese und Pluralitiat. (Münstersche Historische Forschungen, Bd. 5.) Köln/Weimar/Wien 1994. Eine kompakte Darstellung der wissenschaftlichen Bruchlinien im historischen Diskurs um 1900 hat kürzlich Hans Schleier. Historisches Denken in der Krise der Kultur. Fachhistorie, Kulturgeschichte und Anfänge der Kulturwissenschaften in Deutschland. (Essener Kulturwissenschaftliche Vorträge, 7.) Göttingen 2000). vorgelegt. 
mögliche Lösungsansätze skizziere und an einem exemplarischen Fall erprobe. Dabei konzentriere ich mich auf einen Teilaspekt dessen, was individuelle und kollektive Vorstellungen ausmacht, nämlich auf den Aspekt der Wahrnehmung, die hier weniger die verschiedenen Formen sensueller Erfahrung meint, sondern in einem umfassenderen Sinn als der sich kognitiven, affektiven und ethischen Dispositionen verdankende Modus menschlicher Perzeption zu definieren ist.

Daß eine isolierte Betrachtung der Kategorie ,Wahrnehmung ' ein letztlich unmögliches Unterfangen darstellt, ist mir durchaus bewußt. Wenn menschliche Wahrnehmung nicht losgelöst von den kognitiven, affektiven und ethischen Dispositionen, denen sich individuelle und kollektive Vorstellungen verdanken, funktioniert, wirft die Konzentration auf ,Wahrnehmung ' Abgrenzungsprobleme auf und macht zugleich begriffliche Klärungen notwendig. Die Kategorie ,Erfahrung • beispielswcise, die jüngst wieder in einer Reihe von Aufsätzen zur Diskussion gestellt wurde ${ }^{14}$, ist mit der Kategorie ,Wahrnehmung ' untrennbar verbunden. Eine prägnante Unterscheidung der nicht selten synonymisch verwendeten Begriffe ,Wahrnehmung ' und ,Erfahrung * bietet denn auch erhebliche Schwierigkeiten. ${ }^{15}$ Ich habe für meine Darlegungen den Begriff , Wahrnehmung' gewählt, weil er - in, wie ich meine, stärkerem Malße als der Begriff ,Erfahrung - menschliche Perzeption als aktiven und bewußten Akt der Wissenskonstitution und Wirklichkeitsdeutung bereichnet. Er ist außerdem in der methodologischen Diskussion bereits länger in Gebrauch, auch wenn von einer systematischen Auseinandersetzung mit der Kategorie , Wahrnehmung innerhalb des historischen Forschungsdiskurses noch kaum die Rede sein kann. Zwar weist Wahrnehmung in ihrer Eigenschaft als konstitutives Element menschlichen Denkens und Empfindens notwendigerweise Berührungspunkte mit einer Vielzahl von Problemfeldern kulturhistorischer Forschung auf und begegnet als Terminus denn auch in einer Viel\%ahl von Zusammenhängen, ein solides methodisches Fundament wahrnehmungsgeschichtlicher Forschung muß jedoch erst noch

it Vgl. Paul Mänch (Hrsg.). Frfahrung als Kategorie der Frühneuzeitgeschichte. (H\%., Beihefte. NF., Bd. 31.) München 2001.

15 Ähnliches gilt für den Begriff der ,Sensibilität, den Bernd Roeck, Die Wahrnehmung von Symbolen in der Frühen Neuzeit. Sensibilität und Alltag in der Vormoderne, in: Gert Melville (Hrsg.), Institutionalität und Symbolisierung. Verstetigungen kultureller Ordnungsmuster in Vergangenheit und Gegenwart. Köln/Wien/Weimar 2001, 525-539. hier v.a. 527, mit Rückgriff auf Lucien Febvre favorisiert. 
gewonnen werden. ${ }^{16} \mathrm{Da}$ das Problem menschlicher Perzeption sich bis heute nicht als eigenständiger Bereich innerhalb der kulturhistorischen Theoriebildung etablieren konnte, dürfte übrigens nicht zuletzt mit der hier angedeuteten Ubiquität der Kategorie ,Wahrnehmung ' zusammenhängen, die dort, wo ihr Bedeutung zukommt, meist anderen Begriffen subsumiert wird und sich dann zu verflüchtigen droht.

Die Fokussierung auf den Begriff der, Wahrnehmung als ein Kernproblem historischer Methodologie wirft demnach eine Reihe von Fra-

16 Zwar lassen sich eine Reihe von Forschungsbeiträgen nennen, die einer wahrnehmungsgeschichtlichen Perspektive verpflichtet sind, eine systematische Durchdringung der methodischen Probleme, die sich im Zusammenhang mit der Rekonstruktion historischer Perzeptionen stellen, wird darin in der Regel nicht angestrebt. Immerhin thematisieren die nachfolgend genannten Untersuchungen einige zentrale Aspekte und methodische Herausforderungen wahrnehmungsgeschichtlicher Ansïtze. Neben Bernd Roeck (über den bereits in Anmerkung 15 genannten Aufsat $z$ hinaus sind hier noch zwei weitere Beiträge zur Hexenforschung zu erwähnen: Wahrnehmungsgeschichtliche Aspekte des Hexenwahns - Ein Versuch, in: HJb 112, 1992, 72-103, sowie: Säkularisierung als Desensibilisierung. Der Hexenwahn aus der Perspektive der Sensibilitätsgeschichte, in: Sönke Lorenz/Dieter R. Bauer [Hrsg.|, Das Ende der Hexenverfolgung. |Hexenforschung, I.] Stuttgart 1995, 169182), sollen hier die Monographien von Alain Corbin, Pesthauch und Blütenduft. Eine Geschichte des Geruchs. Berlin 1984 (frz. 1982), ders., Les cloches de la terre. Paysage sonore et culture sensible dans les campagnes au XIXe siècle. Paris 2000, Martin Burckhardt, Metamorphosen von Raum und Zeit. Eine Geschichte der Wahrnehmung. Frankfurt am Main/New York 1994, sowie vor allem Robert Jïtte, Geschichte der Sinne. Von der Antike bis zum Cyberspace. München 2000, genannt werden. Einer wahrnehmungsgeschichtlichen Perspektive sind auch die Beitriage in Martin Rheinheimer (Hrsg.), Subjektive Welten. Wahrnehmung und Identität in der Neuzeit. (Studien zur Wirtschafts- und Sozialgeschichte Schleswig-Holsteins, 30.) Neumünster 1998, verpflichtet. Grundsätzlichere Überlegungen finden sich im sorgfältig abwägenden frühen Beitrag von Hans-Werner Goetz, „Vorstellungsgeschichte“. Menschliche Vorstellungen und Meinungen als Dimension der Vergangenheit. Bemerkungen zu cinem jüngeren Arbeitsfeld der Geschichtswissenschaft als Beitrag zu einer Methodik der Quellenauswertung, in: AKG 61, 1979, 253-271, sowic in Vera Nünning, Wahrnchmung und Wirklichkeit. Perspektiven einer konstruktivistischen Geistesgeschichte, in: Gebhard Rusch/Siegfried J. Schmidt (Hrsg.), Konstruktivismus: Geschichte und Anwendung. (Delfin, 1992.) Frankfurt am Main 1992, 91-118. Beachtung verdient in unserem Zusammenhang auch der Beitrag zu frühneuzeitlichen Kometenflugblätlern von Franz Maue/shagen. [llustricrte Flugblätter in wahmehmungsgeschichtlicher Perpektive, in: Wolfgang Harms/Michael Schilling (Hrsg.), Das illustrierte Flugblatt in der Kultur der Frïhen Neuzeit. Wolfenbiitteler Arbeitsgespräch 1997. (Mikrokosmos. Beiträge zur Literaturwissenschaft und Bedeutungsforschung. 50.) Frankfurt am Main 1998, $101-136$. 
gen auf und erfolgt denn auch primär aus heuristischen Gründen: Durch die Konzentration auf einen Teilaspekt einer umfassenderen Problematik sollen die im folgenden formulierten Überlegungen größere Tiefenschärfe und Prägnanz erreichen. Es ist das hier zum Ausdruck gebrachte Bemühen um eine konzise Argumentation, das mich bewogen hat, auf die Integration von in anderen disziplinären Zusammenhängen gewonnenen Erkenntnissen zu verzichten. Die Kategorie ,Wahrnehmung 'spielt nicht nur in der geschichtlichen Reflexion eine Rolle, sie ist auch und immer wieder Gegenstand kognitionspsychologischer ${ }^{17}$, neurobiologischer ${ }^{18}$, physikalischer ${ }^{19}$, philosophischer ${ }^{20}$, kunsthistorischer $^{21}$ oder soziologischer ${ }^{22}$ Untersuchungen gewesen. Wer sich die

17 Vgl. beispielsweise das Standardwerk von John R. Anderson, Kognitive Psychologie. Übers. u. hrsg. v. Ralf Graf u. Joachim Grabowski. 3. Aufl. Heidelberg/Berlin 2001.

18 Für Geschichtswissenschaftler erhellend ist insbesondere der Eröffnungsvortrag auf dem 43. Deutschen Historikertag von Wolf Singer, Wahrnehmen, Erinnern, Vergessen, in: Max Werner (Hrsg.), Eine Welt - Eine Geschichte? 43. Deutscher Historikertag in Aachen 26. bis 29. September 2000. Berichtsband. München 2001, 18-27. Vgl. auch Gerhard Roth, Hirnforschung als Geisteswissenschaft, in: Martin Huber/Gerhard Lauer (Hrsg.), Nach der Sozialgeschichte. Konzepte für eine Literaturwissenschaft zwischen Historischer Anthropologic, Kulturgeschichte und Medientheorie. Tübingen 2000, 29-46.

${ }^{19}$ Die (nicht nur) physikalischen Aspekte optischer Perzeption beschreibt beispielsweise Richard L. Gregory, Eye and Brain. The Psychology of Secing. 5. Aufl. Oxford 1998.

${ }^{20}$ Philosophische Untersuchungen zur Kategorie der Wahrnehmung konzentrieren sich in der Regel aul spezifische Autoren brw. philosophische Richtungen, so bejspielsweise Silvia Stollers Monographie zu Maurice Merleau-Pontys „Phänomenologie der Wahrnehmung“" (Wahrnehmung bei Merleau-Ponty. Studie zur Phänomenologie der Wahrnehmung. |Europäische Hochschulschriften, Rh. 20, Bd. 485.| Bern/Frankfurt am Main/New York (u. a.] 1995), Arkadiusz Chrudzimskis Auseinandersetzung mit Roman Ingardens erkenntnis- und wahrnehmungstheoretischen Schriften (Die Erkenntnistheoric von Roman Ingarden. [Phaenomenologica, 151.] Dordrecht/Boston/London 1999), oder Thomas Blumes Dissertation über die Wahrnehmungstheorie des wissenschaftlichen Realismus (Wahrnehmung und Geist. Über die Einheit von Alltag und Naturwissenschaften. Paderborn 2001).

2I Als Meilenstein in der kunstwissenschaftlichen Erforschung historischer Perzeptionen kann Michael Baxandalls Studie zu den Sehgewohnheiten in der italienischen Renaissance gelten (Die Wirklichkeit der Bilder. Malerei und Erfahrung im Italien des 15. Jahrhunderts. Aus d. Engl. v. Hans-Günter Holl. Frankfurt am Main 1977 |engl. 1972|). Vg! auch Wolfgang Kemp (Hrsg.), Der Betrachter ist im Bild. Kunstwissenschaft und Rezeptionsästhetik. Berlin/Hamburg 1992.

22 So beispielsweise Ashim Hahn. Erfahrung und Begriff. Zur Konzeption einer soziologischen Fifahrungswissenschaft als Beispielhermeneutik. Frankfurt am Main 
Aufgabe stellt, methodische Konturen einer Wahrnehmungsgeschichte zu umreißen, dem bietet sich die Möglichkeit, die in den genannten Disziplinen und Forschungsfeldern erarbeiteten Ergebnisse für seine Fragestellung fruchtbar zu machen. Ein konsequent transdisziplinäres Vorgehen hätte nun allerdings den Rahmen dieses Beitrags gesprengt, und so bleiben die folgenden Ausführungen auf eine geschichtswissenschaftliche Perspektive beschränkt.

II.

Die Erforschung intellektueller und affektiver Dispositionen von Individuen und Kollektiven und damit verbunden die Erforschung menschlicher Wahrnehmung bildet im Kontext einer theoretischen Selbstvergewisserung kulturgeschichtlich ausgerichteter Autoren zwar seit jeher ein zentrales Postulat; eine systematische Analyse historisch manifester Vorstellungs- und Deutungsmuster hat jedoch erst die französische Mentalitätengeschichte initiiert. ${ }^{23}$ Ungeachtet terminologischer Unklarheiten und vielfältiger Divergenzen zwischen den unterschiedlichen Ansätzen, die ihr zugerechnet werden, erlauben es deren Bemühungen, einige zentrale Probleme wahrnehmungsgeschichtlicher Forschung präziser zu beschreiben: Die definitorischen Annäherungen an

1994. Besonders erhellende Einsichten sind der Wissenssoziologie zu verdanken: Peter L. Berger/Thomas Luckmann. Die gesellschaftliche Konstruktion der Wirklichkeit. Eine Theorie der Wissenssoziologie. Übers. v. Monika Plessner. Frankfurt am Main 1969, haben in aller Deutlichkeit herausgestellt, in welchem Maße Wahrnehmung in einen sozialen Kontext eingebettet ist, aus dem heraus sie erklärt werden muls.

23. Die Literatur zur französischen Mentalitätengeschichte hat mittlerweile beeindruckende Ausmaße angenommen. Begriffsklärungen sowic einen konzisen wissenschaftsgeschichtlichen Überblick und eine knappe Darstellung zentraler Postulate der Annales-Historiographie bietet Ute Daniel, Kompendium Kulturgeschichte. Theorien, Praxis, Schlüsselwörter. Frankfurt am Main 2001, 221-232. Textanthologien mit teilweise informativen Einleitungen haben vorgelegt: Claudia Honegger (Hrsg.), Schrift und Materie der Geschichte. Vorschläge zur systematischen Ancignung historischer Prozesse. Frankfurt am Main 1977; Francesco Pitoc'o (Ed.), Storia delle mentalità. I: Interpretazioni. Rom 1996; sowie Matthias Middell/Steffen Sammler (Hrsg.), Alles Gewordene hat Geschichte. Die Schule der ANNALES in ihren Texten 1929-1992. Mit ein. Essay v. Peter Schöttler. Leipzig 1994. Zentralen thematischen Komplexen, zu deren Frforschung die Historiker der Annales Wesentliches beigetragen haben, ist der Band von Peter Dinzelbacher (Hrsg.). Europäische Mentalitätengeschichte. Hauptthemen in Einzeldarstellungen. Stuttgart 1993, gewidmet. 
eine histoire des mentalités verbinden sich seit ihren Anfängen mit einer dezidierten Abgrenzung von der in Deutschland als Geistes- oder Ideengeschichte geiäufigen histoire intellectuelle. Eine kritische Denunziation erfährt letztere zunächst durch Lucien Febvre, welcher der ideengeschichtlich ausgerichteten Historiographie vorwirft, sie richte ihren Blick nicht auf die historischen Einzelphänomene, sondern postuliere die Existenz von Ideen und Denksystemen, welche jenseits der konkreten menschlichen Erfahrung in einer Sphäre gedanklicher Abstraktion ihren Ort tänden. Damit würden Ideen als geschichtsmächtige Kräfte aus ihren historischen Zusammenhängen isoliert. Sie schwebten gewissermaßen über dem geschichtlichen Prozeß, seien greifbar nur in den durch herausragende Individuen geschaffenen literarischen, philosophischen und künstlerischen Artefakten, nicht jedoch in den historisch spezifischen Lebenswelten unterschiedlicher Epochen. ${ }^{24}$ Gegen ein derartiges Verständnis geistiger Prozesse wenden sich auch einige französische Historiker, die in der seit 1929 erscheinenden Zeitschrift „Annales d'histoire économique et sociale" publizieren, indem sie den eher unscharfen Begriff , Mentalität ' cinführen und damit eine Reihe im Laufe der Zeit sich wandelnder theoretischer Forderungen verbinden: Fundamental für die Auffassung von Geschichte als Mentalitätengeschichte ist crstens die Überzeugung, daß Mentalitäten nicht adäquat erforscht werden können, wenn sich die Analyse auf Denksysteme und Wissenshorizonte einer kulturellen Elite beschränkt. An gesellschaftlicher Sinnproduktion partizipieren alle sozialen Gruppen; die Untersuchung individueller und kollektiver Vorstellungswelten muß 3 deshalb auch und gerade jene Gruppen umfassen, die, nicht zuletzt aufgrund mangelhafter Quellenüberlieferung, in der älteren Forschung weitgehend ausgeblendet wurden. Mentalitäten interessieren zweitens als $k o l$ lektive soziokulturelle Muster. Anders als in der Ideengeschichte, in der die Selbstoffenbarungen von Individuen gewissermaken als pars pro toto die Existen\% und den historischen Wandel von geistigen Dispositionen belegen sollen, sind es weniger die singulären Auffassungen herausragender Subjekte als vielmehr die gemeinschaftlichen Vorstellungen größerer Gruppen, die es zu erfassen gilt. Im Unterschied zur Ideengeschichte, die ihren Blick auf einige wenige sich im geschicht-

24 Vgl. daru Roger Chartier, Intellektuelle Geschichte und Geschichte der Mentalitäten, in: Ulrich Raulff (Hrsg.), Mentalitäten-Geschichte. Zur Rekonstruktion geistiger Prozesse. Berlin 1989.69-96. hier 71-77. 
lichen Ablauf sukzessive herausbildende und fortschreitende geistige Konzepte richtet, beharren die Historiker der Annales drittens auf der Forderung, jede Epoche als autonome Entität zu betrachten. Innerhalb räumlich und zeitlich voneinander abgegrenzter Einheiten bilden sich je eigene intellektuelle und affektive Konfigurationen, die nur im Rahmen einer sorgfältigen Rekonstruktion epochenspezifischer Vorstellungsmodi zu erschließen sind. Kennzeichnend für die in einem Zeitraum dominierenden Mentalitäten ist viertens nicht nur, daß sie kollektiv in Erscheinung treten, sondern auch, daß sie das Handeln historischer Subjekte ohne deren Wissen bestimmen. Während die Ideengeschichte geistige Konfigurationen als bewußte Konstruktionen eines exemplarischen Individuums versteht, zielt die Mentalitätengeschichte auf jene Vorstellungen, die keiner expliziten Formulierung und unmittelbaren Manifestation bedurften, um sich dennoch als geschichtsmächtig zu erweisen.

Den Annales-Historikern verdanken wir nicht nur eine in Auseinandersetzung mit der histoire intellectuelle gewonnene Neubestimmung dessen, was eine auf die historischen Vorstellungswelten von Menschen gerichtete Historiographie leisten könnte, sondern auch ein methodisches Instrumentarium, das es erlauben soll, kollektive Mentalitäten auf wissenschaftlich plausible Weise zu beschreiben. Zu nennen sind einerseits die quantifizierenden Methoden der histoire sérielle ${ }^{25}$ und andererseits die auf der Grundlage eines zahlenmäßig begrenzten, jedoch besonders aussagekräftigen Quellenkorpus argumentierende qualitative Mentalitätenforschung. Das sich serieller Quellen bedienende quantifizierende Verfahren, für das Michel Vovelles 1970 erschienene Studie „La piété baroque et la déchristianisation en Provence", in der er anhand mehrerer tausend von Verstorbenen hinterlassener Testamente das Frömmigkeitsverhalten im südlichen Frankreich des 18. Jahrhunderts untersucht, geradezu beispielhaft steht, geriet seit den frühen 1970er Jahren zunehmend in die Kritik und wurde in der Folge zuriickgedrängt von den immer zahlreicheren qualitativen Forschungen einer jüngeren Generation von Historikern, dic sich der histoire nouvelle zuordnen. ${ }^{26}$

25 Yum methodischen Instrumentarium der histoire sérielle vgl. J'an-Michel Thirief, Methoden der Mentalitätsforschung in der französischen Sozialgeschichte. in: Ethnologia Europaea 11, 1979/80. 208-225.

26. Fine Antwort auf die Kritik an der histoire sériclle versucht Michel Vovelle in einem 1985 erstmals erschienenen Beitrag (Serielle Geschichte oder, case studies": 
Die Verdienste, aber auch die theoretischen Defizite und Irrwege der histoire des mentalités hat Roger Chartier, auch er ein Vertreter der Annales-Tradition, auf besonders scharfsinnige und für die hier interessierende Fragestellung weiterführende Weise reflektiert. Es würde nun allerdings zu weit führen, Chartiers Kritik an der französischen Mentalitätengeschichte umfassend zu referieren. In unserem Zusammenhang scheinen mir insbesondere zwei methodische Einwände bedeutsam: Der erste zielt auf die Art und Weise, wie nicht wenige Exponenten der Annales-Schule das Verhältnis zwischen Quelle und darin zum Ausdruck gelangender historischer Wirklichkeit definieren, der zweite auf die Vernachlässigung der zeitgenössischen Praktiken, die den Umgang mit jenen Zeugnissen, die der Historiker retrospektiv zur Quelle erhebt, bestimmen. ${ }^{27}$ Problematisch erscheint Chartier eine Auffassung, die in Quellen unmittelbare Abbildungen der Wirklichkeit erkennt. Kein Text unterhält. so Chartier, eine transparente Beziehung zur Wirklichkeit, die er thematisiert. Dic Beziehung des Textes zur Realität ist vielmehr nach ,diskursiven Modcllen“ gestaltet, die ,jeder Schreibsituation eigen sind" 28 , der Text bildet kein eineindeutiges Abbild, sondern ein vieldeutiges Konstrukt wirklicher Erfahrung, ein Konstrukt, dessen spezifische Beschaffenheit sich ebenso sehr individuellen wie kulturellen Vorgaben verdankt. Neben den spezifischen Intentionen des jeweiligen Verfassers sind es vor allem literarische Konventionen, welche die Konfiguration eines Schriftwerks determinieren. Der einzelne Text folgt grammatikalischen und stilistischen Regeln. ist rhetorischen und ästhetischen Vorgaben verpflichtet, fügt sich ein in die formale Matrix,

ein wirkliches oder nur ein Schein-Dilemma? in: Raulff [Hrsg.], Mentalitäten-Geschichte [wie Anm. 24]. 114-126). Unter den zahlreichen qualitativ verfahrenden Studien, die repräsentative schriftliche. aber auch bildliche Quellen heranzichen, um jeweils das kulturelle Deutungs- und Wertesystem einer spezifischen Gruppe innerhalb eines begrenzlen historischen Zcitraums zu rekonstruieren. hat Emmanuel Le Roy Laduries auf Inquisitionsakten basierende Analyse des Lebensalltags und der Vorstellungs- und Gefühlswelt der Bewohner eines Pyrenäendorfs um die Wende vont 13. zum 14. Jahrhundert besondere Berühmtheit erlang1 (Montaillou. Ein Dorf vor dem Inquisitor 1294 bis 1324. Frankfurt am Main 1980 [frz. 1975]). ${ }_{27}$ Dic folgenden Ausführungen stiitzen sich vor allem auf zwei Publikationen Roger Chartiers: Kulturgeschichte zwischen Repräsentationen und Praktiken. in: ders., Die unvollendete Vergangenheit. Geschichte und die Macht der Weltauslegung. Berlin 1989. 7 . 20, sowie den Aufsat\%: Die Welt als Repräsentation, in: Middell/Sammler (Hrsg.). Alles (iewordene hat Geschichte (wie Anm. 23), 320)-347, worin die darin vertretene Position erläutert und differenziert wird.

28 ('hatter. Geschichte der Mentalitäten (wie Ann. 24), 91 f. 
an die er durch seine Gattungszugehörigkeit gebunden ist. Gleichzeitig gilt es zu bedenken, daß Texte medial vermittelt werden, daß sie beispielsweise als Buch, als Zeitungsartikel, als Pamphlet, als Zeitschriftenaufsatz oder als Kalenderbeitrag konkrete Gestalt gewinnen. Chartier warnt folgerichtig davor, den materiellen Charakter von Druckerzeugnissen zu ignorieren. Nur durch einen Zugang, der textanalytische Verfahren mit buchgeschichtlicher Forschung verbindet, könnten die vielfältigen Bedeutungs- und Wirkungsmöglichkeiten textueller Repräsentationen adäquat erfaßt werden. Inwiefern und auf welche Weise nun das Bedeutungspotential eines Textes eine konkrete Realisierung erfährt, hängt von dessen Rezeption ab. Die Sinnproduktion eines Textes erfolgt in engster Abhängigkeit von einem Individuum oder einem Kollektiv, das diesen Text wahrnimmt. Textbedeutung ist demnach weder autonom noch statisch, sie wird vielmehr durch Leser generiert, die in spezifische historische Kontexte eingebettet sind. Zwar spielt die formale und materiale Beschaffenheit des Textes eine entscheidende Rolle für die aus ihm zu gewinnenden Wahrnehmungen, indem sie Lesarten ermöglicht, aber auch Lesarten verhindert, dennoch gilt, daß Bedeutung erst im Akt des Rezipierens konstituiert wird. ${ }^{29}$ Signifikanz kommt demzufolge in erster Linie jenen kulturellen Praktiken zu, welche nicht nur die Genese, sondern auch und vor allem den zeitgenössischen Umgang mit einer Quelle bestimmen. ${ }^{30}$ So plädiert

${ }^{29}$ Chartier, Welt als Repraisentation (wic Anm. 27), 335, verweist in diesem Zusammenhang zu Recht auf die deutsche Rezeptionsästhetik, moniert jedoch, daß diese zwar auf dic Bedeutung der Rezeption für die literarische Sinnkonstitution hingewiesen habe, jedoch die materielle Basis der Kommunikation zwischen Text und Leser vernachlässige: Letztere erscheint als ,reine und unmittelbare" Bezichung zwischen den vom Text ausgehenden, Signalen` und dem ,Erwartungshorizont ' der Rezipienten, die für den Leseprozel relevante Form, in der ein Text gedruckt erscheint, wird völlig ausgeblendet.

30) Der Rekonstruktion historischer Leseweisen hat Chartier mehrere Untersuchungen gewidmet, die teilweise in deutscher Übersetzung vorliegen: Roger Chartier, Lesewelten. Buch und Lektüre in der frühen Neuzeit. Aus d. Franz. v. Brita Schleinitz u. Ruthard Stäblein. (Historische Studien, 1.) Frankfurt am Main/New York/ Paris 1990. Vgl. dazu dic Besprechung von Haiko Wandhof, Der Medienwechsel als Epochenschwelle? Roger Chartiers Studien zur Geschichte des Lesens in der frühen Neureit, in: Zs. für Germanistik NF. 6, 1996, 76-83. Vgl. auch Roger Chartier. „Volkstümliche“ Leser und ihr Lesestoff von der Renaissance bis zum Age classique, in: Nada Boškovska Leimgruber (Hrsg.), Dic Frühe Neuzcit in der Geschichtswissenschaft. Forschungstendenzen und Forschungserträge. Paderborn/ München/Wien//ürich 1997, 229-247. Über die Frühe Neuzcit hinaus reicht der 
Chartier für eine Kulturgeschichte, die der, Aneignung “ als Gegenstand wissenschaftlicher Reflexion eine zentrale Rolle zuweist und die jene „Analyse der Praktiken“ leistet, welche deutlich werden läßt, wie sich Individuen und Kollektive ,auf verschiedene Weise der symbolischen Güter bemächtigen und unterschiedliche Verwendungen und Bedeutungen produzieren". ${ }^{31}$ Die konkreten Modalitäten des Leseprozesses, die Normen, welche den Lesevorgang steuern, gilt es zu beachten; zugleich und vor allem jedoch müssen jene sozialen und kulturellen Dispositionen in den Blick genommen werden, welche im Rahmen individueller und kollektiver Wahrnehmung die Sinnkonstitution steuern. Dabei genügt es nicht, von angeblich a priori gegebenen sozialen $\mathrm{Ge}-$ gensätzen ausgehend, zwischen Eliten und Volk zu trennen, wie dies die ältere Mentalitätengeschichte bisweilen getan hat ${ }^{32}$; ebensowenig taugt die von Michel Foucault vorgeschlagene Unterscheidung zwischen den die Diskurse beherrschenden Institutionen und Individuen einerseits und den der Diskursgewalt ausgelieferten einzelnen und Gruppen andererseits ${ }^{33}$. Beide Positionen unterstellen nämlich die Existenz eines von kulturellen Eliten verwalteten Herrschaftswissens, das all jenen, die nicht zu diesen Eliten gehören, auf gezielte Art und Weise vermittelt oder auch vorenthalten wird. ,Aneignung" im Sinne Chartiers bedeutet nicht einfach die Verinnerlichung autoritativ vorgegebener Deutungsmuster, sondern bezeichnet einen ,multilateralen`InteraktionsprozeßB, an dem alle Beteiligten aktiv partizipieren. Die aus diesem Prozeß3 resultierenden individuellen Aneignungsmodi sind folgerichtig nur zum Teil das Ergebnis durch Eliten gelenkter Steuerungsmechanismen, sie verdanken sich in nicht geringerem Maße Parametern wie beispielsweise Geschlechts- oder Generationszugehörigkeit, religiösen Überzeugungen, Bildungserfahrungen, regionalen Verankerungen oder beruflichen Gewohnheiten. ${ }^{34}$ Die Rekonstruktion historischer Wahrnehmungen hat dem/ufolge nicht isoliert zu crfolgen, sie soll vielmehr angebunden werden an eine ..Sorialgeschichte der Interpretation vor dem Hintergrund ihrer fundamentalen Determinationen (die gesellschaftlicher, institutioneller. kultureller Art sind) und in ihrer Einbet-

Sammelband: Roger ( Hertier/Gugliclmo (arallo (Eds.). Histoire de la lecture dans le monde occidental. Rom/3ari 1995.

3 (hartier. Welt als Reprisisentation (wie Anm, 27), 328.

i2 Vol. cbd. 332.

is Vol. chd. 331 .

if Vgl. ched. 3,32 . 
tung in spezifische Praktiken, die sie hervorbringen". 35 Chartiers in kritischer Auseinandersetzung mit der Tradition der Annales gewonnenes Konzept einer Kulturgeschichte, die der menschlichen Perzeption und den mit ihr verbundenen kulturellen Praktiken oberste Priorität einräumt, bildet meines Erachtens einen geeigneten Ausgangspunkt für die Darlegung einiger grundsätzlicher Probleme wahrnehmungsgeschichtlicher Forschung. Ein Historiker, der sich mit vergangenem Geschehen befaßt, hat keinen unmittelbaren Zugang zum Gegenstand seiner wissenschaftlichen Bemühungen, denn Vergangenheit ist nur greifbar in den Spuren, welche sie hinterlassen hat. Wer sich demnach die Aufgabe stellt, einen spezifischen geschichtlichen Moment hinsichtlich der in ihm belegbaren Wahrnehmungsmuster zu untersuchen, sieht sich auf jene Quellen verwiesen, die zeitgenössische Perzeptionsmodi dokumentieren. Diese Quellen nun stehen in einem intrikaten, für den Historiker nur bedingt durchschaubaren Verhältnis zur Wirklichkeit, die sie repräsentieren. Sie reproduzieren nicht, Realität', sondern stellen das Ergebnis komplexer Wahrnehmungsoperationen dar, aus denen sich die jeweils in einer Quelle konstituierte, Wirklichkeit ${ }^{6}$ ergibt. ${ }^{36}$ Sie bilden außerdem keine isolierten Erscheinungen, sondern stehen in einem kommunikativen Zusammenhang, werden - dies gilt insbesondere für die gedruckte Überlieferung - in der Regel bereits von zeitgenössischen Rezipienten wahrgenommen und gedeutet. Unter wahrnehmungsgeschichtlicher Perspektive sind nicht nur jene, primären' Perzeptionen eines Phänomens bedeutsam, welche sich aus einer Quelle erschließen lassen, sondern auch die je unterschiedlichen, sckundären " Wahrnehmungen, welche durch den zeitgenössischen Gebrauch einer Quelle entstehen. Das Erkenntnisinteresse des Kulturhistorikers hat sich demzufolge, um mit Roger Chartier zu sprechen, einerseits auf die ,Repräsentationen" menschlicher Vorstellung und andererseits auf die

35 Chartier, Kulturgeschichte zwischen Repräsentationen und Praktiken (wie Anm. 27), 18 .

36 Auch Hans-Werner Goet-, Vorstellungsgeschichte (wie Anm. 16). 256, weist darauf hin, daß eine, Vorstellungsgeschichte' nicht, die Vergangenheit in ihrer Faktizität [rekonstruiert], sondern die Vergangenheit als die verarbeitete Wirklichkeit des Zeitgenossen" ". Noch radikaler konstruktivistisch verfährt Nïnning, Wahrnehmung und Wirklichkeit (wie Anm. 16), 92, wenn sie die .,Welt des Menschen" ausschließlich als .,das Produkt seiner Konstruktionen“" definiert und deutlich macht, dal3 historische Überlieferung keinen Zugang zu einer hinter den menschlichen Wahrnehmungen stehenden, Realitiat bietet. sondem bestenfalls individuelle und kollektive Konstruktionen von . Realität erschließ3t. 
zeitgenössischen ,Praktiken` der Aneignung, die sich mit diesen Repräsentationen verbinden, zu richten. Dies ist umso wichtiger, als eine Quelle nie nur eine Lesart zuläßt, sondern immer unterschiedliche Wahrnehmungen dessen, was sie in Wort und bisweilen auch im Bild zum Ausdruck bringt, ermöglicht. Die zumindest für Philologen seit jeher selbstverständliche Einsicht, daß kulturelle Artefakte hinsichtlich ihres Sinns nicht eindeutig festgelegt sind, daß sie vielmehr ein System von Zeichen konstituieren, welches eine allerdings begrenzte Zahl unterschiedlicher Interpretationen zuläßt, führt dazu, daß man mit Blick auf Quellen als Objekte von zeitgenössischer Wahrnehmung das diesen inhärente Wahrnehmungspotential beschreiben kann, nicht jedoch eine einzige der jeweiligen Quelle angemessene Perzeptionsweise. Nicht nur hinsichtlich der, Wahrnehmungsobjekte', auch in bezug auf die ,Wahrnehmungssubjekte' herrscht keine Eindeutigkeit. Ein und dasselbe Artefakt kann zu je verschiedenen Zeitpunkten, von je verschiedenen Individuen und Gruppen auf unterschiedliche Weise wahrgenommen werden. Die historische Erforschung der Kategorie ,Wahrnehmung ' kann von dieser Variabilität menschlicher Perzeption nicht absehen. Sie hat im Gegenteil die heterogenen individuellen und gemeinschaftlichen Perzeptionsmodi, die im Zusammenhang mit einem Artefakt nachweisbar sind, so umfassend wie möglich in den Blick zu nehmen, um so der Diskontinuität und Diskordanz historischer Wahrnehmungen Rechnung zu tragen. ${ }^{37}$ Indem sie jedoch ihr Augenmerk gleichermaßen auf Quellen als ,Repräsentationen“ historischer Erfahrung und auf die mit diesen verknüptten kommunikativen Praktiken richtet, erschließ3t sie sich eine zusätzliche Untersuchungsebene, die es ermöglicht, das weite Feld sich eröffnender Deutungsoptionen wieder einzugrenzen und die bisweilen disparaten geschichtlichen Relikte adäquater einzuordnen. Dic Rekonstruktion von Kommunikationszusammenhängen, innerhalb derer Quellen zeitgenössisch rezipiert werden, lenkt den Blick auf die Steuerungsmechanismen und die Autorisierungsstrategien, denen Wahrnehmungen in sperifischen geschichtlichen Zeiten und Räumen unterworfen sind, und erlaubt es, Vorstellungen zu beschreiben, welche die Wahrnehmung von Individuen und Gruppen über soziale oder religiöse Grenzen hinweg dominieren, und

37 Chartier. Welt als Repräsentation (wie Anm. 27), 342 f., betont die Notwendigkeit. die Vorstellung in sich homogener Mentalitaten und damit verbunden Wahrnehmungsmuster zu verabschieden und den Blick zu schärfen für dic ..Diskontinuität" und "Diskordan/" historischer Vorstellungswelten. 
damit zu unterscheiden zwischen dem, was mit Blick auf einen historischen Moment als kulturelle Norm bzw. als Abweichung definiert werden kann.

Illustriert werden soll das vorgängig Erörterte durch einen exemplarischen ,Fall': Es handelt sich um ein am 10. September 1580 in weiten Teilen Mitteleuropas sichtbares Polarlicht, das durch eine bemerkenswert dichte Überlieferung publizistischer und chronikalischer Quellen bezeugt ist und deshalb besonders geeignet erscheint, individuelle und kollektive Perzeptionen natürlicher Phänomene in der Frühen Neuzeit zu erhellen. Eine umfassende Analyse der Wissensbestände, Affektlagen und religiös-moralischen Normsysteme, welche die in den untersuchten Quellen zutage tretenden Wahrnehmungsmodi determinieren, können und wollen die folgenden knappen Ausführungen nicht leisten. Die Aufmerksamkeit gilt primär der Frage, was sich mit Blick auf das hier interessierende geophysikalische Phänomen über die Wahrnehmung breiterer Bevölkerungsschichten in Erfahrung bringen läßt. Im Rahmen einer synchronen Betrachtung - Quellen zu früheren und späteren ähnlichen Ereignissen bleiben unberücksichtigt - soll das bisher Dargelegte erprobt, sollen jene vorläufigen Postulate fundiert werden, dic ich abschließend zur Diskussion stellen will.

\section{III.}

Wohl noch im Jahre 1580 veröffentlicht der Augsburger „Brieffmaler“ Bartholomäus Käppeler ${ }^{38}$ ein Flugblatt, auf dem ein ,,sehr erschröcklichs Wunderzeychen/ so man im Jahr 1580. den 10. September/ in der Keyserlichen Reichstatt Augspurg/ nach vndergang der Sonnen/an dem Himel" gesehen habe, in Wort und Bild dargestellt ist. ${ }^{39}$ Der kolo-

\footnotetext{
38 Von Bartholomäus Käppeler sind nur wenige Drucke überliefert: Neben Einblattdrucken zum Kometen vom 12. November 1577 und zur Belagerung Mühlhausens im Jahr 1587, zwei Prodigienblättern und einem Porträt des Fürsten Sigismund Báthory sind zwej Flugschriften über die Ermordung des französischen Königs Heinrich III. (1589) und der Belagerung Temesvárs (1596) durch Sigismund Báthory erhalten. Vgl. Bruno Weber (Hrsg.), Wunderzeichen und Winkeldrucker 1543-1586. Einblattdrucke aus der Sammlung Wickiana in der Zentralbibliothek Zürich. Dictikon-Zürich 1972, 125.

${ }^{39}$ Das Flugblatt ist abgedruckt und kommentiert in Wolfgang Harms/Michael Schilling (Hrsg.), Dic Sammlung der Zentralbibliothek Zürich. Kommentierte Ausgabe. T. 2: Dic Wickiana II (1570) 1588). (Deutsche illustrierte Flugblätter des 16. und 17. Jahrhunderts, Bd. 7.) Tübingen 1997. Nr. 131.
} 


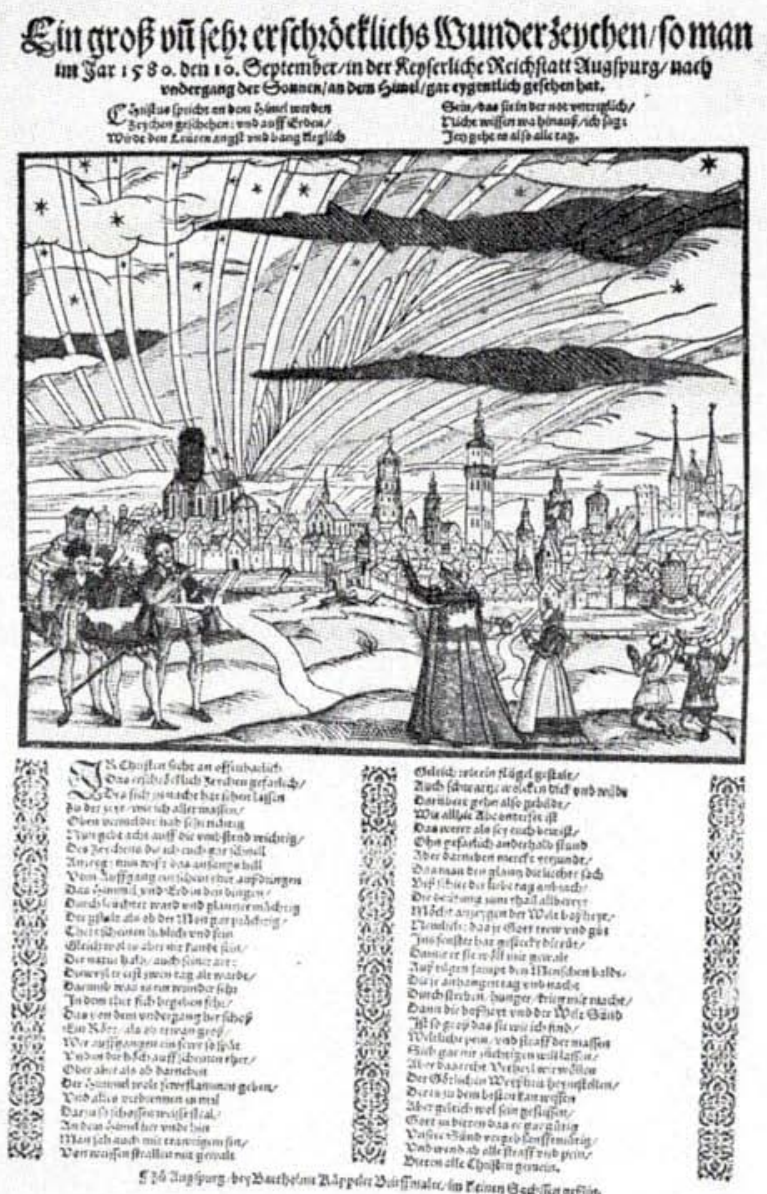

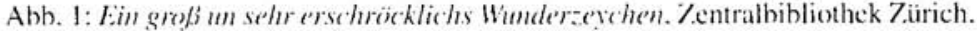
PAS II 17/13.

rierte Ilolzschnitl ( $\Lambda$ bb. 1) zeigt eine Ansicht der Stadt Augsburg. über der sich am nächtlichen Himmel jene unerklärliche Erscheinung abspielt. welche dic im Vordergrund betindlichen Figuren betrachten. Dic ausführliche Beschreibung des Phänomens im Text dürfte nicht zulet/九t damit zusammenhängen, dal3 die H limmelserscheinung - es handelt sich um ein Polarlicht - die zeitgenössischen Beobachter mit erheb- 
lichen klassifikatorischen Schwierigkeiten konfrontierte. ${ }^{40}$ Anders als etwa bei den Kometen, stellte die Einordnung von Polarlichtern den frühneuzeitlichen Betrachter sowohl in wissenschaftlicher als auch in theologischer Hinsicht vor offenkundige Probleme. Die antiken naturkundlichen Theorien in der aristotelischen Tradition ordneten diese nur allgemein den Himmelserscheinungen, den Chasmata, $\mathrm{zu}^{41}$, was dem Verfasser des vorliegenden Blatts ebensowenig bekannt gewesen sein dürfte wie die Tatsache, daß bereits 1561 eine aus der Feder des Züricher Gelehrten Conrad Gessner stammende „Historia et Interpretatio Prodigii“" erschienen war, in der erstmals ein Nordlicht wissenschaftlich beschrieben wurde ${ }^{42}$, betont er doch wiederholt den widernatürlichen Charakter der Erscheinung ${ }^{43}$. Nicht ohne Bedeutung dürfte außer-

40 Zum Phänomen des Polarlichts aus wissenschaftsgeschichtlicher Perspektive vgl. Wilfried Schröder, Das Phänomen des Polarlichts. Geschichtsschreibung, Forschungsergebnisse und Probleme. (Erträge der Forschung, 218.) Darmstadt 1984, und neuerdings Dirk Tölke, Polarlichter, in: Hans Holländer (Hrsg.), Erkenntnis, Erfindung, Konstruktion. Studien zur Bildgeschichte von Naturwissenschaften und Technik vom 16. bis zum 19. Jahrhundert. Berlin 2000, 463-482.

${ }^{41} \mathrm{Zu}$ antiken Polarlichtbeschreibungen vgl. Schröder, Phänomen des Polarlichts (wie Anm. 40), 9-16. Schröder weist allerdings darauf hin, daß die uneinheitliche Terminologie es bisweilen erschwert zu bestimmen, welche meteorologisch-physikalische Erscheinung die aus der Antike überlieferten Darstellungen von Himmelsphänomenen beschreiben (ebd. 12).

42 Wem wir die erste gedruckte Polarlichtbeschreibung zu verdanken haben, ist umstritten. Während Gustav Hellmann, Die Meteorologie in den deutschen Flugschriften und Flugblättern des 16. Jahrhunderts. Ein Beitrag zur Geschichte der Meteorologie. (Abhandlungen der Preußischen Akademie der Wissenschaften, Physikalisch-mathematische Klasse, Jg. 1921, 1.) Berlin 1921, 36, davon ausgeht, dußs es sich bei Peter Creutzers 1527 als Flugschrift erschienener Beschreibung eines, „Cometen“ in Wahrheit um die Darstellung eines Polarlichts handelt, nimmt Schröder, Phänomen des Polarlichts (wie Anm. 40), 24-27, mit plausiblen Argumenten an, daß Conrad Gessners „Historia et Interpretatio Prodigii“ die erste in Druck erschienene und von cinem wissenschaftlichen Erkenntnisinteresse ausqehende Beschrcibung cines Polarlichts enthält. $\mathrm{Zu}$ Gessners Polarlichtbeschrcibung vgl. Thomas Gutwald, PRODIGIUM HOC CUM NOSTRO SECULO INUSITATUM SIT... Das Nordlicht vom 28. Dezember 1560 als Gegenstand vernetater Wahrnehmungen durch frünneuzeitliche Informationssysteme, in: Wahrnehmungsgeschichte und Wissensdiskurs im illustrierten Flugblatt der Frühen Neuzeit (1450-1700). Hrsg. v. Wolfgang Harms, Alfred Messerli in Verb. mit Frieder von Ammon u. Nikola von Merveldt. Basel 2002, 239-261, hicr 250-257.

4.3 Zur Unterscheidung zwischen natürlichen' und , unnatürlichen', d.h. götlichen Zeichen vil. Barbara Bauer, Dic Krise der Reformation. Johann Jacob Wicks Chronik außergewöhnlicher Natur- und Himmelserscheinungen, in: Harms/Mes- 
dem der Umstand gewesen sein, daß Polarlichter im Gegensatz zu anderen astronomischen oder geophysikalischen Erscheinungen in der Bibel an keiner Stelle als Zeichen des nahenden Weltendes erwähnt werden, was deren heilsgeschichtliche Deutung erschwerte. Ungeachtet der hier genannten Schwierigkeiten gelangt der Verfasser des Flugblatts zu einer Interpretation des Phänomens und stellt zugleich dem Leser bzw. Betrachter eine Reihe unterschiedlicher Sinnstiftungsangebote zur Verfügung, von denen ich hier vier nennen möchte: Dem naturkundlich interessierten Rezipienten vermittelt das Flugblatt Informationen zur Beschaffenheit der Himmelserscheinung. In Übereinstimmung mit der bildlichen Darstellung berichtet der Verfasser, wie zunächst von Osten her ein sehr helles Licht den Himmel erleuchtet habe, bevor aus dem Westen eine feuerähnliche „Röte“ aufgestiegen sei, die den Eindruck entstehen ließ, der Himmel stünde in Flammen. Vor diesem Hintergrund seicn weiße Strahlen ,her vnde hin“ geschossen, durchbrochen von länglichen schwarzen Wolken. Das Schauspiel habe ungefähr eineinhalb Stunden gedauert, dessen Nachwirkungen seien allerdings noch fast bis zur Morgendämmerung am Himmel sichtbar gewesen. Die Beschreibung des Polarlichts nun wird ergänzt durch eine theologische Auslegung des Phänomens. Bereits in der ersten Zeile wird an alle „Christen“ appelliert, dem ,erschröcklichen Zeychen" die ihm gebührende Aufmerksamkeit zu schenken. Dessen Deutung dominiert die zweite Hälfte des Textes. Die „boßheyt“ und die „,sünd“ der Welt hätten den Höchsten dazu bewogen, ,die rùt ins fenster" $z u$ stecken und damit zu signalisieren, daß den Menschen ein göttliches Strafgericht bevorstünde. Folgerichtig schliel.3t der Verfasser seine Darstellung mit der Aufforderung, ,Gott zu bitten das er gar gütig Vnsere Sünd vergeb sanfftmütig/ Vnd wend ab alle straff vnd pein." Der Aufruf zur Buße wiederum verbindel sich mit dem Hinweis auf „,sterben", ,hunger" und „krieg“ als den Konsequenzen menschlicher Sündhaftigkeit und bringt damit eine dritte mögliche Lesart ins Spiel: Das Prodigium verweist nicht nur auf einen transzendenten Bereich, sondern auch auf die geschichtliche Welt, indem es den Blick des Betrachters auf Epidemien. Hungersnöte und Kricge als konkrete Erfahrungen frühneuzeitlicher Menschen lenkt. ${ }^{44}$ Wenn schließ3lich betont

serli (Hrsg.). Wahrnehmungsgeschichte und Wissensdiskurs (wic Anm. 42). 19.3 236, hier $211-214$.

it 'Zur Bedeutung von . Pestilen/". Teuerung" und , Krieg' als den drei , Hauptplitgen der Menschheit in der Darstellung und Interpretation von Himmelserscheinun- 
wird, das ,erschröckliche“ Himmelszeichen bewirke, daß „,den Leüten angst vnd bang" wird, scheint eine vierte, affektbeherrschte Rezeptionsweise auf. 45

Der vorliegende Einblattdruck enthält zwar in Text und Bild eine Reihe hier nicht im einzelnen zu erläuternder rezeptionssteuernder Elemente, er ermöglicht dem Betrachter und Leser dennoch unterschiedliche Wahrnehmungs- und Deutungsmöglichkeiten, die dieser einzeln oder auch kombiniert realisieren konnte. Es stellt sich nun allerdings die Frage, welche der hier beschriebenen Sinnstiftungsangebote von zeitgenössischen Rezipienten aufgegriffen wurden und auf welche Weise dies geschah. Einen ersten Hinweis scheint das Flugblat selbst zu liefern, stellt es doch im Vordergrund mehrere Figuren dar, die gewissermaßen prototypisch mögliche Reaktionen auf das Geschehen veranschaulichen. Die Körperhaltung, vor allem jedoch die Anordnung der drei männlichen Figuren links im Bild läßt erkennen, daß sie im Begriff sind, sich über das merkwürdige Phänomen zu verständigen, es zu reflektieren und zu diskutieren. Kennzeichnend für die zentrale weibliche Figur ist der ausgeprägte Zeigegestus, der sich nicht nur dadurch erklären läßt, daß sie sich einer Mädchenfigur zuwendet. In die Mitte der Graphik gerückt, bildet sie eine personifizierte Aufforderung an den Betrachter, das Dargestellte mit derselben Aufmerksamkeit wahrzunehmen wie die abgebildeten Augenzeugen. Die beiden betenden bzw. die Hände gegen den Himmel erhebenden Knabenfiguren rechts im Bild schließlich führen jene Gottesfurcht und Bußfertigkeit vor Augen, zu welcher der Text aufruft. aber auch die Angst, welche die ungewöhnliche Erscheinung bei den Betrachtern auslöst. Wie auch der Text lenkt die Illustration demnach den Blick auf unterschiedliche Wahrnehmungsmodi und gibt keinen hinreichenden Aufschluß ïber konkrete historische Wahrnehmungen der unerklärlichen Himmelserscheinung. Um individuelle Perzeptionen, aber auch kollektive Wahrnehmungs-

gen sgl. Wiebke. Schurarte. Nordlichter. Ihre Darstellung in der Wickianal. Münster/ Now York/München/Berlin 1999. 71. 77.

t5 Zur Funktion der in lrühneuzeitlicher Bildpublizistik wiederholt begegnenden Fpithela schrecklich", wunderlich". grausam" vgl. Alfred Messerli. Angst und Wunderreichen in Einblatldrucken. Überlegungen zu einem Bild/lext-Medium aus der zweiten Hälfte des 16. Jalhrhunderts. in: Rolf Wilhelm Brednich/Andreas Hartmann (Hrsg.). Populäre Bildmedien. Vortrüge des 2. Symposiums für ethnologische Bildforschung Reinhtusen bei Göttingen 1980. (Schriftenteihe der Volkskundlichen Kommission für Viedersachsen. Bd. 4.) Göttingen 1989. 131-150. 
muster des hier interessierenden geophysikalischen Phänomens zu rekonstruieren, bedarf es demnach weiterer Zugänge. Da die Instrumente empirischer Rezeptionsforschung uns nicht zur Verfügung stehen - die zu befragenden Zeitzeugen sind längst gestorben -, ist es notwendig, zum einen zusätzliche zeitgenössische Quellen auf darin sich manifestierende Darstellungs- und Deutungsweisen von Polarlichtern hin zu befragen und zum andern mit Blick auf das vorgängig erörterte Flugblatt nach überlieferten Rezeptionsspuren zu suchen.

Eine Beschreibung des Polarlichts vom 10. September 1580 findet sich auch in der handschriftlichen Chronik des protestantischen Augsburger Handelsdieners Georg Kölderer ${ }^{46}$ : Ein „Erschröckhlich, Wunderbarlich vnnd Mörckhlich gesicht" sei an besagtem Tag um neun Uhr abends am Himmel zu erkennen gewesen. ${ }^{47}$ Der Chronist wei $\beta$ in der Folge zu berichten, daß3 der Himmel ,ganntz Hell vnnd Liecht“ geworden sei, ,gleichsam der Mon Scheinendt“ und daß sich „Inn sollicher erscheinenden Liechte [...] alls baldt vill grosser Weysser strüemmen, oder stralen (sich gegen vnd Inn Mitte des Aufgangs vnd Mittnacht Streckhend) sehen lassen“. Die weilsen Strahlen hätten sich daraufhin rot verfärbt, seien in "gestallt grosser feurflammen" sichtbar gewesen, so dass man ,Ausserhalb der Statt, vermaint gehabtt, das ein grosse brunst bey vnns Auskhommen were". Wie auch der Autor des Flugblatts „Ein groß vnd schr erschröcklichs Wunderzeychen“ erwähnt Kölderer dunkle Wolkenformationen, spricht von einem ,gewölckh, gleich einer grossen Rauch von einer Brunst" und weist darauf hin, daß die Erscheinung fast die ganze Nacht zu beobachten gewesen sei.48 Nicht nur hinsichtlich der Beschreibung des Phänomens, sondern auch hinsichtlich seiner Auslegung gibt es zwischen dem Einblattdruck aus Käppelers Offizin und Kölderers Aufzeichnungen bemerkenswerte Ühereinstimmungen. So betont Kölderer, Gott habe das Himmelszei-

4h \%ur Chronik des Georg Kölderer vgl. Benedikt Maurer, , Gemain Geschrey und .teglich Reden". (jeorg Kölderer " ein Augshurger Chronist des konfessionellen Zcitalters. (Veröffentlichungen der Schwäbischen Forschungsgemeinschaft, I/29).) Augsburg 2001. Die Kölderer-Chronik befindet sich in der Statats- und Stadtbibliothek Augsburg: der hier interessierende erste Band trägt die Signatur $2^{\circ}$ Cod. S. 39. Fine kommentierte Ldition der Chronik ist in Vorbereitung, sie dürfte voraussichtlich 2005 erscheinen. Eine Transkription der im folgenden zitierten Polarlichtbeschreibung wurde mir freundlicherweise won der Bearbeiterin. Frau Dr. Silvia Strodel. zur Verfügung gestellt.

17 Chronik Geor: Kolderer (wie Anm. 46), fol. 79 v.

tr Find. 
chen „Zusonnderlicher warnung sehen lassen, auff das wir doch einmall vmb kheretten, von Sünden zu der Buess, das verleych der Liebe Gott durch Ihesum Christum, vmb welliches willen vnnd Allerhailligste fürbitt, Er seinen Gerechten, vnd billichen Zoren vnnd straffen, von vns genedigclich wöll abwennden" 49 , und fügt seinem Bericht einen gereimten Text bei, in welchem die religiöse Bedeutung von Prodigien herausgestellt wird ${ }^{50}$. Wenn darin Epidemien (,Khranckhaitt") und politisch-militärische Konflikte („An Allen Khünigreichen Mechtig, Wie die zergeen, werden zwittrechtig Vnnd zerthrennet sich als geleich, durch aus Inn Aller Welltte Reich") angekündigt werden, gerät auch die profane Erfahrung von Menschen in den Blick, und das Insistieren auf dem ,schröckhlichen“ Charakter des Phänomens offenbart die Ängste, welche das Polarlicht im Chronisten erzeugt hat.

Noch erhellender als Georg Kölderers Bericht ist die ebenfalls ungedruckte Chronik des Zürcher Chorherrn Johannes Wick. ${ }^{51}$ In ihr findet

49 Ebd.

${ }^{50}$ Es spricht viel dafür, daß das in die Chronik integrierte ,Gedicht " einem Flugblatt entnommen wurde. Die Suche nach einer allfälligen Vorlage blieb bisher allerdings ergebnislos. Der vollständige Text lautet: „Dises Iar hatt vnns Gott gethrew, vill Wunderzaichen Seltzam New, Sehen Lassen, merckht Auff vnnd Hörtt Fürnemlich, am Gewechs der Erdt, Am Hümell, vnnd dem Fürmament, Am Menschen, Khranckhaitt vnerkhent, An Allen Khünigreichen Mechtig, Wie die zergeen, werden zwittrechtig Vnnd zerthrennet sich als geleich, durch aus Inn Aller Welltte Reich. Die Hertzen der Menschen Ietzundt, Nicht Mainen Recht, aus guettem grundt Einannder wie sy Pillich solltten, Sonnder villieber haben wolltten, das Alles was das annder hätt, es nur Inn seim sackh steckhen thette Ain Annder gonnt man Hässlich nit Das Ains die Son An schein mit fridt, So gar ist man Ainannder g'hass Vnnd sündigett Immerhin fürbass. Niemandt Kerett von sünden vmb, vnnd vervrsacht das Gott darumb Schickhett Souill der Warnungs Zaichen Das sy vnns zu der buess erwaichen. Aber man Schlecht es Alls Inn Wündt Vermaint nit das Gott strafft die Sündt, Fertt fort In den selben mit gefar Vnnd wann Gott hett In disem Iar Noch souill Zaichen geschechen liess Weniger mann sich daran stiess. So Gar khain Gottesforcht bey den Leuith Ist bey den Leüthen dieser Zeitt, Gott mach vnns zu der Buess bereitt. Amen" (Chronik Georg Kölderer [wie Anm. 46], fol. 8()$r)$.

51 Zu Johannes Wick und seiner Chronik vgl. Mathias Senn, Johann Jakob Wick (1522-1588) und scine Sammlung von Nachrichten 7ur Zeitgeschichte. Diss, phil. Zürich 1973, sowic Schwarte, Nordlichter (wic Anm. 44), 2-10 u. 30-35. Von Matthias Senn stammt auch eine kommentierte Anthologie mit Texten und Bildern aus der Wickschen Chronik (Die Wickiana. Johann Jakob Wicks Nachrichtensammlung aus dem 16. Jahrhundert. Texte und Bilder zu den Jahren 1560) bis 1571 . Küsnacht/Zürich 1975). Eine frühe Beschreibung der Wickschen Nachrichtensammlung verdanken wir Ricarda Huch. Die Wicksche Sammlung von Flugblättern und 
sich nicht nur eine ausführliche Darstellung des Phänomens aus der Sicht des Chronisten, sondern auch das oben beschriebene Flugblatt, mithin ein Nebeneinander von handschriftlicher Primärquelle und gedruckter Publikation und damit zugleich eine aufschlußreiche Rezeptionsspur. ${ }^{52}$ Der Augsburger Einblattdruck über das Polarlicht vom 10. September 1580 ist im Anschluß an einen Augenzeugenbericht eingeheftet, der beschreibt, wie die Himmelserscheinung sich in Zürich manifestierte. Bemerkenswert ist nun, daß die handschriftlichen Eintragungen die Wahrnehmungs- und Deutungsangebote des Flugblatts umfassend realisieren. Der naturkundliche Blick des Chronisten zeigt sich in der detaillierten Beschreibung des Polarlichts, die durch Überlegungen zu meteora, also Himmelserscheinungen aus natürlichen Ursachen, zu denen allerdings das Nordlicht vom 10. September 1580 nicht zu zählen sei, ergänzt werden. Auch in Johannes Wicks Chronik erscheint das Phänomen nämlich als göttliches Zeichen, als ,ein sunderbare verkündung, und ein ernstliche warnung vor künfftigen ellend und großem jammer".53 Im Rahmen einer realhistorischen Interpretation findet das Prodigium im Gesamtzusammenhang der Chronik allerdings eine noch konkretere Deutung: Die Polarlichterscheinung, wie die Häufung von

Zeitungsnachrichten in der Stadtbibliothek Zürich, in: dies., Gesammelte Werke. Hrsg. v. Wilhelm Emrich. Bd. 9. Köln/Berlin 1968, 261-304. Spezicll mit den in die Chronik eingehefteten Flugblättern befaß3t sich Weber, Wunderzeichen und Winkeldrucker (wie Anm. 38). Bruno Weber ist auch eine Faksimile-Edition ausgewählter Flugblätter aus der Wickiana zu verdanken (Erschröckliche und warhafftige Wunderzeichen 1543-1586. Faksimiledruck von Einblattdrucken aus der Sammlung Wikiana in der Zentralbibliothek Zürich. Dietikon/Zürich 1971); das bei Bartholomäus Käppeler erschienene Flugblatt über die Polarlichterscheinung vom 10. September 1580 ist dort reproduziert (Tafel 17). Eine vollständige kommenticrte Edition der Wickiana-Einblattdrucke wird unter der Leitung von Wolfgang Harms vorbereitet. Der Band mit den Flugblättern der Jahre 1570)-1588 ist 1997 erschienen (wie Anm. 39), die Publikation des Bandes, der die vor 1570 gedruckten Flugblätter der Wickiana enthält, steht unmittelbar bevor.

52 Angesichts der Tatsache, daß3 Wick als Chronist und als Sammler den Prodigien besondere Aufmerksamkeit geschenkı hat, ist es nicht verwunderlich, daß dessen Auseinandersetzung mit Himmelserscheinungen in der Forschung auf Interesse gestoßen ist. Vgl. Schwarte, Nordlichter (wie Anm. 44), und zuletzt Bater, Krise der Reformation (wie Anm. 43).

53 Zitiert nach Barbara Bauer, Kommentar zu Warhafftige ind erschrö̈kliche Newe Zeyttung, in: Harms/Schilling (Hrsg.), Wickiana (wie Anm. 39), Nr. 139 |S. 280|. Die Beschreibung des Nordlichts vom 10. September 1580 in der Wickschen Chronik ist abgedruckt in Schwarle, Nordlichter (wie Anm. 44). 131-134, hier 132. 
Himmelszeichen überhaupt, setzt der reformierte Geistliche Wick in seinen Aufzeichnungen wiederholt in Bezug zu der sich verschärfenden Verfolgung der Hugenotten seit den Massakern der Bartholomäusnacht. ${ }^{54}$ Das Gefühl von Bedrohung und Angst schließlich, welche das Ereignis auch ausgelöst hat, artikuliert sich in der Rekurrenz von Termini wie „erschrokenlich". 55

In Johannes Wicks Chronik begegnet uns demnach ein Rezipient, der sich die durch den vorgängig erörterten Einblattdruck bereitgestellten Wahrnehmungsmuster zu eigen macht und auf komplexe Weise in Relation zueinander setzt. ${ }^{56}$ Fraglich ist nun allerdings, welche Repräsentativität eine derartige Perzeption für sich beanspruchen kann, gilt es doch zu bedenken, daß im 16. Jahrhundert nur eine Minderheit der Bevölkerung über den Bildungshorizont des Züricher Geistlichen und Gelehrten verfügte und diesen außerdem bei der Abfassung seiner Chronik ganz individuelle Intentionen geleitet haben dürften. Immerhin hat ein Blick in die andersgeartete Chronik Georg Kölderers gezeigt, daß sich auch in diesem Fall die Beschreibung des Phänomens mit dessen religiöser Nutzanwendung sowie dem Hinweis auf zu erwartende Seuchen sowie politisch-militärische Umwälzungen verbindet und daß aus den Formulierungen jene Affektlage spricht, die auch in der Wickschen Chronik begegnet. Um ein umfassenderes Bild historisch belegbarer Wahrnehmungsangebote und konkreter Perzeptionen zeichnen zu können, bedarf es der Integration weiterer Quellen. Durch die Einbeziehung von Selbstzeugnissen wie private Korrespondenz oder Diarien würde es möglich, zusätzliche individuelle Perzeptionen mit der chronikalischen Überlieferung zu vergleichen; durch die Berücksichtigung zeitgenössischer naturwissenschaftlicher und insbesondere astronomischer Schriften ließe sich die gelehrte Auseinandersetzung mit Himmelserscheinungen rekonstruicren. ${ }^{57}$ Damit sind jedoch erst mögliche

54 In welchem Maße der reformierte Geistliche Wick die Häufung von Prodigien mit den konfessionellen Auseinandersetzungen seiner Zeit in Verbindung bringt, hat Baller, Krise der Reformation (wie Anm. 43), herausgestellt.

55 Vol. Schwarte, Nordlichter (wic Anm. 44), $132 \mathrm{f}$.

56 Auch Bauer, Krise der Reformation (wie Anm. 43), 236, beobachtet, da13 Johannes Wick und seine Korrespondenten sich ,im Spiegel ihrer Wunderzeichenberichte als Leser [präsentieren], die ziemlich genau dem Bild des Rezipienten entsprechen, welchen die Finblattdrucke ansprechen".

57 Dic Auseinandersetzung mit und um Himmelserscheinungen erfolgl zu einem nicht unwesentlichen Teil in den zahlreich publizierten Flugschriften und Flugblättern zu astronomischen und geophysikalischen Phänomenen (vgl. I/el/mamn. Me- 
Perzeptionsmodi von Individuen, welche den politischen, kirchlichen und kulturellen Eliten zuzurechnen sind, erfaßt; wie der ,gemeine Mann ${ }^{58}$, der in der frühneuzeitlichen Bildpublizistik wiederholt als Adressat angesprochen wird, eine Nordlichterscheinung wie diejenige des Jahres 1580 wahrgenommen hat, ist so noch nicht geklärt. Hierzu bedarf es der Einbeziehung zusätzlicher Quellenkorpora wie zum Beispiel gedruckter Predigten, Prodigienliteratur oder frühneuzeitlicher Kalender, die breiteren Bevölkerungsschichten zugänglich waren und wesentlich zu einer Popularisierung naturkundlichen Wissens beigetragen haben dürften. Im folgenden konzentriere ich mich auf die Predigt, weil sie mir besonders geeignet erscheint, Einblick in mögliche Wahrnehmungsmodi illiterater bzw. nicht alphabetisierter Individuen und Gruppen zu gewähren.

Die meisten gedruckten Predigten, die eine Himmelserscheinung zum Anlaß haben, sind als Flugschrift erschienen. Dies gilt auch für Jacob Colers zu Beginn des Jahres 1581 veröffentlichte Predigt über das Polarlicht vom 10. September 1580. ${ }^{59}$ Die Predigt beginnt mit einer de-

teorologie [wie Anm. 42], und Schröder, Phänomen des Polarlichts [wie Anm. 40], 19-38), reicht jedoch auch in dic gelehrten Korrespondenznetze und findet - vereinzelt im 16. und dann vor allem seit dem frühen 17. Jahrhundert - Ausdruck in gelehrten Druckwerken (vgl. Schröder, Phänomen des Polarlichts [wie Anm. 40], 37-46, sowie Tolke, Polarlichter [wie Anm. 40], 472-475). Auf welches Interesse die Erforschung kosmischer und terrestrischer Erscheinungen auch und gerade in der Reichsstadt Augsburg stießB, belegen die Aktivitäten des späthumanistischen Kreises um den Augsburger Patrizier Marcus Welser. Zu Welsers wissenschaftlichem Netzwerk und dessen publizistischen Bemühungen vgl. Inge Keil, Markus Welser und die Naturwissenschaften, in: Mark Häberlein/Johannes Burkhardt (Hrsg.), Die Welser. Neuc Forschungen zur Geschichte und Kultur des oberdeutschen Handelshauses. (Colloquia Augustana, 16.) Berlin 2002, 609-617.

${ }^{58}$ Zum Begriff des, gemeinen Mannes“ im 16. und 17. Jahrhundert vgl. Robert $H$. Lutz. Wer war der gemeine Mann? Der dritte Stand in der Krise des Spätmittclalters. München/Wien 1979.

59) Der vollständige Titel der Flugschrift lautet: Notwendige Erinnerung/ auff d7. schreckliche Fewerzeichen/ So den 10. Septembris deß3 jetrlatuflenden 1580. Jahrs am Himmel gesehen worden/ sampt ciner gründlichen gewissen Rechnung aus Gottes Wort/ dali das Ende der Welt vnd der Jüngste lag nahe für der Thüren IACOBVS COLERVS D. stamus in occursum cupida tibi mente parati: Quando tibi visum est, CHRISTE, venire, veni. l uce am 21 Capitel. Sehet an den Fejgenbaum vnd alle Beume/ wenn sie jetzt aubschlahen/ so sehet jhrs an jhnen vnnd mercket/ daß jetzt der Sommer nahe ist. Also auch jhr/ wenn jhr dilb alles sehet angehen/ so wisset/ dalB das Reich Gottes nahe ist/ etc. Gedruckl zu Berlin/ Anno 1581. Ein Exemplar der Flugschrift befindet sich in der Bayerischen Staatsbibliothek München. 
taillierten Beschreibung des Phänomens ${ }^{60}$ : Bei Sonnenuntergang hätten sich am Himmel kleine rote Wolken gezeigt, die in der Folge aus Osten von einer großen hellen Wolke überstrahlt worden seien, welche das gesamte mittlerweile verdunkelte Firmament erleuchtete. Aus der Wolke seien weiße Strahlen hin und her geschossen. Nach dem Verschwinden der hellen Wolke, habe sich eine mächtige rote Wolke gebildet, die dem Betrachter ,wie ein grosser rodter fewriger lenglicher Hewschober/ der lichter lohe gebrand hette" (Bijv) erschien. Die Wolke habe daraufhin jene adlerartige Form angenommen, die eine Vignette auf dem Titelblatt der Flugschrift darstellt. Nachdem sich die Wolkenformationen verzogen hätten, sei am Himmel, ein weißer ,Zirckel“" aus Licht, d. h. eine Corona, sichtbar gewesen, von dem Lichtblitze ausgingen. Die an die Beschreibung anschließende Auslegung des Polarlichts macht deutlich, daß dem evangelischen Geistlichen die diskursiven $\mathrm{Zu}-$ sammenhänge, in denen sich die zeitgenössische Auseinandersetzung mit Himmelserscheinungen bewegt, nicht fremd sind: „Nu weis ich gewiß/ gelehrte Leute werdens für kein Wunder/ sonder für ein natürlich $\mathrm{ding} /$ vnd für ein Meteoron/ daß man Chasma pflegt zunennen/ halten/ vnd werden sagen/ es bedeute grosse dürre vnnd heisse Zeitten/ daraus grosse Tewrung kommen kan/ vnd wenn ich nicht ein Christ/ sondern ein Heide were/ vnnd hette kein ander Buch denn Aristotelem, Plinium, Pontanum vnd dergleichen Authores, vnd wüste von dem rechten waren Gott vnd der Bibel nichts/müste ich warlich selber sagen/ es were ein Chasma, vnd were nicht ein fixum, sondern Volubile, Mobile, oder Variabile, wie die Heiden vnd Naturkunder von den dingen pflegen zu reden" (Biijv). Die „Naturkunder" stehen hier für jene sich auf antike Autoren berufenden Gelehrten, die auf dem natürlichen Ursprung von Polarlichtern beharren und diesen, indem sie einen influxus physicus der supralunaren Sphäre auf die sublunare Welt postulieren, natürliche

Es trägt die Signatur Res. $4^{\circ}$ Astr. P. 528-13. Nach diesem Exemplar wird im folgenden zitiert. Während Hellmann, Meteorologie (wic Anm. 42), zum Polarlicht vom 10. September 1580 ein einziges Flugblatt - es handelt sich um den aus der Oftizin Käppelers stammenden Einblattdruck - nachweist, nennt er gleich mehrere Flugschriften, in denen das hier interessierende Phänomen beschrieben und gedeutet wird: Neben zwei Flugschriften, die im Rahmen einer Darstellung verschiedener, Wunderzeichen' auch auf das besagte Nordlicht Bezug nehmen, verweist Hellmann auf den Colerschen Predigtdruck sowic auf eine Flugschrift aus der Feder des evangelischen Theologen Peter Rauner (vgl. ebd. 79 f.).

60) Die Beschreibung ist abgedruckt in Weber. Wunderzeichen und Winkeldrucker (wie Anm. 38), $123 \mathrm{f}$. 
Wirkungen zuschreiben. ${ }^{61}$ Coler distanziert sich explizit von einer derartigen Auffassung und begründet seine Haltung damit, daß das unregelmäßige Auftreten derartiger Erscheinungen und deren variable Gestalt gegen einen natürlichen Ursprung spreche, denn „die Natur wircket nicht ungleich ding/ sondern allezeit einerley/ vnd wens nicht einerley wircket/ so ists nicht natürlich ding/ sondern eitel Gottes wunder" (Biiijr). Im übrigen hätten, wie Coler mit Hinweis auf Livius betont, auch die antiken Autoren Himmelserscheinungen als ,prodigia" bezeichnet und damit ihren göttlichen Ursprung bestätigt. ${ }^{62}$ Als von Gott ausgehendes Zeichen nun bedarf das beobachtete Polarlicht einer adäquaten Lektüre. Colers Interpretation geht von der beschriebenen adlerartigen Wolkenformation aus. Den Adler deutet er als Sinnbild des Römischen Reichs, dessen Ende in greifbare Nähe gerückt sei. Um seine Aussage zu stützen, erinnert der Theologe an jene in biblischer und patristischer Überlieferung wurzelnden Periodisierungssysteme, mit Hilfe derer noch bis weit ins 17. Jahrhundert der universalhistorische Ablauf gegliedert wurde. ${ }^{63}$ Mit Hilfe sowohl des auf Daniel 2, 31-45 zurückgehenden Modells der vier Monarchien als auch der durch Augustinus verfochtenen Lehre von den sechs Weltaltern sei es möglich zu belegen, daß die Apokalypse unmittelbar bevorstünde. Dafür sprächen auch jene Zeichen der Endzeit, die Coler in Anlehnung an Luther in der Folge benennt. Colers Erkenntnis lautet denn auch: „Summa/ Der Seiger ist vbel gestalt/ die Laute vbel bezogen/ keine

6l Die auf Aristoteles und Ptolemaios zurückgehende ,metcorologjsche "Wirkungstheorie. die besagte, daß Gestirne (meteora) auf den irdischen Mikrokosmos wirkten, indem sic als Katalysatoren von Dürse und daraus resultierend Hungersnöten fungierten und den Tod von Herrschern sowie Kriege herbeizuführen vermochten, war unter frühneuzeitlichen Gelehrten populär (vgl. John D. North, Celestial Influence - the Major Premiss of Astrology, in: Paola Zambelli [Ed.], ,Astrologi hallucinati'. Stars and the End of the World in Luther's Time. Berlin/New York 1986, 45-100). Da der terrestrische Charakter von Polarlichtern erst spät erkannt wurde, konnten ihnen in der Frühen Neuzeit dieselben Wirkungen zugcschricben werden wie den Kometen

$62 \mathrm{Vgl}$. Coler, Notwendige Erinnerung (wic Anm. 58), Biiijv.

6.3 $\mathrm{Zu}$ den die frühneuzeitliche Geschichtsauffassung prägenden Periodisierungssystemen vgl. Silvia Serena Tschopp. Heilsgeschichtliche Deutungsmuster in der Publizistik des DreiBigjährigen Krieges. Pro- und antischwedische Propaganda in Deutschland 1628 his 1635. (Mikrokosmos, 29.) Frankfurt am Main 1991, 277287. Ausführlicher Wilhelm Schmidt-Biggemann, Philosophia perennis. Historische Umrisse abendländischer Spiritualitäl in Antike, Mittelalter und Früher Neuzeit. Franklurt am Main 1998. 585-.64.5. 
Seite concordiret mit der andern/ es ist ein grewlich geheul vnnd ein böser Gesang in allen Landen [... ] Die Welt wil eingehn/ sie kracht an allen örten/ sie muß brechen vnd einfallen/ denn sie kan sich nicht lenger halten“ (Dv). Vor diesem Hintergrund erscheint das Himmelszeichen vom 10. September 1580 als göttliche Mahnung: Aufgabe jedes Christen sei es, sich durch Sündenbekenntnis und Buße auf das Jüngste Gericht vorzubereiten, um vor dem göttlichen Richtstuhl zu bestehen und damit das ewige Heil zu gewinnen. Bereits in der Widmungsvorrede, in der er sein Werk drei weiblichen „Kirchkindern“ (Aiiijv) zueignet, bemüht sich Coler um eine spirituelle Deutung des Polarlichts und betont in diesem Zusammenhang, mit dem Prodigium wolle Gott ,anzeigen $[\ldots], \mathrm{da} ß$ er von wegen vieler Sünde vnnd Schande sein strenges Gericht vber die Welt nicht lenger kan auffhalten/sondern bald kommen werde/ vnd es mit der Welt ein ende machen" (Aiijr). Nachdem er zur geistlichen Besinnung aufgerufen und an das göttliche Gnadenangebot erinnert hat, kann der lutherische Theologe seine Predigt mit den zuversichtlichen Worten schließen: „Wir wollen gern für dein Gericht kommen/ sintemal wir gründlich wissen/ daß du vns eine gute Herberg bereitet hast/ da wir bey dir Ewiglich sein vnd bleiben sollen/ Amen/ Amen/ Kom lieber HERR Jesu Christe/ vnd hole vns bald heim/ vnd bleib ja nicht lang aussen/ Amen" (Eijr). Gottes Walten wird nun allerdings nicht cinfach in den Bereich zukünftiger Erwartung verbannt, es manifestiert sich bereits in gegenwärtiger Erfahrung. Außergewöhnliche Phänomene verweisen, wic Coler wiederholt hervorhebt, nicht nur auf das ans Ende der Geschichte gerückte göttliche Weltgericht, sie antizipieren zugleich zeichenhaft irdische Katastrophen. So stellt der Berliner Geistliche das Polarlicht nicht nur in einen heilsgeschichtlichen Kontext, sondern erkennt Zusammenhänge zwischen der Himmelserscheinung und den zeitgenössischen politisch-militärischen Ereignissen, insbesondere dem Krieg um Livland, und integriert damit auch eine profangeschichtliche Perspektive.

Bartholomäus Käppelers Einblattdruck, Georg Kölderers Aufzeichnungen, Johannes Wicks Chronik sowie Jacob Colers Flugschrift zur Polarlichterscheinung vom 10. September 1580 erweisen sich als in mehrfacher Hinsicht aufschlußreich. Zum einen sind sie ein weiterer Beleg dafür, in welchem Malie insbesondere in protestantischen Territorien die Wahrnehmung der eigenen Gegenwart eschatologisch fundiert war. Sowohl der reformierte Chorherr Wick als auch der lutherische Geistliche Coler leben in der zeittypischen Überzeugung, die Pa- 
rusie stehe unmittelbar bevor. ${ }^{64}$ Zahlreiche militärische Konflikte in Europa, die auch nach dem Augsburger Religionsfrieden anhaltenden konfessionellen Spannungen im Reich sowie die vom Osmanischen Reich ausgehende Gefahr, die auch nach der türkischen Niederlage bei Lepanto nicht gebannt war, trugen dazu bei, ein Krisenbewußtsein zu generieren, das die Sensibilität für ungewöhnliche Ereignisse und die Bereitschaft, diese als göttliche Zeichen zu interpretieren, erhöhte. ${ }^{65}$ $\mathrm{Da}$ sich Himmelserscheinungen und insbesondere Polarlichter gegen Ende des 16. Jahrhunderts in auffälliger Weise häuften ${ }^{66}$, dürfte die Zeitgenossen darin bestätigt haben, daß fundamentale Umwälzungen bevorstanden. Nicht zufällig verweist Jacob Coler denn auch auf die Vielzahl von Prodigien und wertet sie als Indiz für kommendes Unheil. ${ }^{67}$

Die bisher untersuchten Quellen bringen nicht nur die im 16. Jahrhundert verbreitete Endzeiterwartung zum Ausdruck, sie erlauben Rückschlüsse auf das zeitgenössische Naturverständnis. Als einer der ersten hat Jürgen Mittelstraß herausgestellt, daß die Herausbildung neuer wissenschaftlicher Paradigmen seit dem 16. Jahrhundert nicht zu einer plötzlichen Verdrängung älterer Konzepte geführt hat. ${ }^{68}$ Ganz. offenkundig dominiert im späten 16. Jahrhundert die auf mittelalterliche Vorstellungen zurückgehende Auffassung des Kosmos als eines

64 Welche Bedeutung dem BewuBstsein des nahenden Weltendes im 16. Jahrhundert beizumessen ist, zeigt Bernd Roeck am Beispiel Augsburgs (Eine Stadt in Krieg und Frieden. Studien zur Geschichte der Reichsstadt Augsburg zwischen Kalenderstreit und Parität. |Schriftenreihe der Historischen Kommission bei der Bayerischen Akademic der Wissenschaften, 37.J Göttingen 1989, 71-87).

$6.5 \mathrm{Vgl}$ dazu dic bercits erwähnte Studie von Bater, Krise der Reformation (wie Anm. 43), sowic Irene Eisinkel, De monstris. Deutung und Funktion von Wundergeburten auf Flugblïttern im Deutschland des 16. Jahrhunderts. (Frühe Neuzeit, 23.) Tübingen 1995 .

to6 Insbesondere für die Jahre 1.571 bis 1583 sind eine Vielzahl von Polarlichtbeschreibungen überliefert (vgl. Hermann Fritz, Verzeichniss !!) beobachteter Polarlichter. Wien 1873. 22 f.). Für das Gebiet der Fidgenossenschaft nennt Hans Fehr, Massenkunst in 16. Jahrhundert. Flugblätter aus der Sammlung Wickiana. Berlin 1924. 53, dic ungewöhnlich hohe Zahl von insgesamt 35 Polarlichterscheinungen allein zwischen 1571 und 1590. Vgl. auch und vor allem Schwarte, Nordlichter (wic Anm. 44), 45-53.

${ }^{67}$ Die entsprechende Stelle lautet: ..Wehe Deutschlandt/ wegen der vielfeltigen Iiewer vnd anderer Zeichen/derer wir bißanhero in grosser Anzahl gesehen/ in allerley lormen vnnd (iestalten." (Coler, Notwendige Erinnerung / wie Anm. 58|, Bijr). of Vgl. Jürgen Miftelstra/3, Neuzeit und Aufklärung. Studien zur Entstehung der neuzitlichen Wissenschaft und Philosophic. Berlin/New York 1970. 
,Buchs', in das Gott seine Botschaften einschreibt. ${ }^{69}$ Die natürliche Welt bildet einen universalen Zeichenzusammenhang, den der Mensch zu entziffern hat, wenn er seine Bestimmung erfahren will; ungewöhnliche Himmelserscheinungen sind folgerichtig als Prodigien, als von Gott gesandte Vorboten künftigen Unheils, zu betrachten. ${ }^{70}$ So überrascht es nicht, daß sich die Urheber der hier interessierenden Quellen gegen jene Auffassungen verwehren, die Polarlichter als natürliche, physikalisch erklärbare Phänomene interpretieren und den göttlichen Ursprung und damit den unnatürlichen Charakter der erörterten Himmelserscheinung betonen. Die Tatsache allerdings, daß in ,Wunderzeichenblättern " und Flugschriften zu Himmelserscheinungen wiederholt beklagt wird, Prodigien würden von vielen Menschen in ihrer Bedeutung unterschätzt, kann auch als Indiz für einen zunehmend skeptischeren Umgang mit derartigen Phänomenen gelesen werden. So heißt es in einer 1582 in Augsburg gedruckten ,Warhafftige[n] vnd erschröckliche[n] Newe[n] Zeyttung " über die Polarlichterscheinung vom 6. März desselben Jahres: „ES hat vnser Herr Gott/ der nit ein Gott ist/ dem Gottloß wesen gefelt/ sonder hasset alle Vbelthäter/ Psal. 5. dise weyß allwegen gehalten/ wann er hat etwas newes wöllen machen/ die Welt entweder zu begnaden oder zustraffen/ das er hat sondere grosse Zaichen lassen vorher gehen: Welliches die Schrifft mit Sprüchen vnd Exempeln bezeuget allenthalben [...]. Also hat er noch heuttigs tags dise seine gewonheit/ laßt vns offt schröckliche Zaichen am Himmel vnd Erden sehen/als lebendige Prediger/damit er vns ja dernmal eins zur rechtgschaffnen Buß bringen künde. Aber (leyder) der mehrer thails keret sich nicht allein gar nichts an solche Zaichen/sonder verachtets darbey mit hönischen worten/ sprechent: Sie seyen vorhin offt gesehen

$69 \mathrm{Vgl}$. Hans Blumenberg, Dic Lesbarkeit der Welt. Frankfurt am Main 1981.

${ }^{70}$ Zum Zeichencharakter natürlicher Phänomene und in diesem Zusammenhang zur Bedeutung von Prodigien in Mittelalter und Früher Neuzeit vgl. Michael Rothmann, Zeichen und Wunder. Vom symbolischen Weltbild zur scientia naturalis, in: Melville (Hrsg.), Institutionalität und Symbolisicrung (wie Anm. 15), 347-392. Vgl. auch Schwarte, Nordlichter (wie Anm. 44), 26-29 (dort Hinweis auf ältere Literatur). Welch herausragende Bedeutung Georg Kölderer - durchaus im Einklang mit seiner Zeit - den Prodigien als göttlichen Zeichen beimißt, kommt in seiner Chronik wiederholt zum Ausdruck. Für Maurer, Georg Kölderer (wie Anm. 45), 329-346. hier 329, ist er denn auch der ,intensivste Augsburger Beobachter von Zeichen - monstra - im gesamten Untersuchungszeitraum“". Johannes Wicks Vorlicbe für, Wunderzeichen " wurde von der Forschung bereits früh bemerkt (vgl. die Literaturhinweise in Anm. 50). 
worden/ es seyen gewönliche Gesichten/ die nichts sonders bedeuten. Wöllens derhalben auch nit recht mit zerknirschtem hertzen anschawen/ sondern schlaffen vnnd schnarchen vil lieber darfür on alle sorge." 71 Auch wenn derartige Äußerungen mit Vorsicht interpretiert werden müssen ${ }^{72}$, verweisen sie auf rationalere Perzeptionsmodi und dokumentieren einen sich seit dem späten 16. Jahrhundert andeutenden Bewußtseinswandel.

Wie die hier berücksichtigten Quellen belegen, schließt die theologische Deutung natürlicher Phänomene deren präzise Erfassung keinesfalls aus. ${ }^{73}$ Es fällt im Gegenteil auf, daß die vorgängig untersuchten Quellen nicht nur eine sehr ausführliche, sondern auch bemerkenswert übereinstimmende Darstellung des Polarlichts vom 10. September 1580 bieten. Darüber hinaus bemühen sich deren Autoren nicht nur um eine detaillierte Beschreibung des sie interessierenden Phänomens, sie betonen im Anschluß an ihren Bericht auch die Glaubwürdigkeit des Dargelegten. So beendet Coler den deskriptiven Teil seiner Ausführungen mit den Worten: „Diß ist fast die beschreibung/ wie ichs gesehen: Andere so auff den Thürmen gestanden/ oder im Felde gewesen/ mögen mehr gesehen haben/ denn wir in der Stadt zwischen den Heusern/ wie ich mir denn derer observationes auch etliche habe auffschreiben lassen/aber aus jren verzeichnussen wenig anders finden können/ denn

71 Das Blatt ist abgebildet und kommentiert in Harms/Schilling (Hrsg.), Wickiana (wie Anm. 39), Nr. 139.

72 Wichke Schwartes These eines „Gewohnheitseffekts“ (Schwarte, Nordlichter [wie Anm. 44], 84), mit dem sich eine skeptischere Haltung Prodigien gegenüber begründen lasse. greift meines Erachtens zu kurz. Schwarte übersieht, daß die sich in Wunderzeichenblättern manifestierende Kritik an der Gleichgültigkeit himmlischen Botschaften gegenüber eine rhetorische Strategie darstellen kann, die sich am adäquatesten mit dem Begriff ,Zweckpessimismus' bezeichnen läßt. Dennoch scheint es plausibel anzunehmen, daß die gegen Ende des 16. Jahrhunderts geradezu topische Klage über die ,Uneinsichtigkeit" mancher Zeitgenossen nicht eines realen Hintergrunds entbehrt.

73 Daß im 16. und 17. Jahrhundert Himmelserscheinungen zugleich mathematicè, physicè vel metheorologicè und theologicè betrachtet werden können, wobei die verschiedenen Perzeptionsmodi gleichberechtigt nebeneinander stehen, stell auch Christian von Zimmermann in scinem Beitrag zu Kometenpredigten heraus (,Wie man den Cometen [...] soll betrachten“. Zwei Predigten des Jahres 1618 aus Riga und Magdeburg im Kontext der frühneuzeitlichen Kometenliteratur, in: Wilhelm Kühlmann/Wolf-Dieter Müller-Jahncke [Hrsg.], Iliaster. Literatur und Naturkunde in der Frühen Neuzeit. Festgabe für Joachim Telle zum 60. Geburtstag. Heidelberg 1999, 321-344, hier 342). 
ich selber vermerckt/ so ist mir auch sicherer von dem/ das ich selber gesehen/ zu reden/ denn in eines andern Kopf zukrichen/ vnd seinen auffmerckungen nach zuahmen."74 Auch Georg Kölderer hält in seiner Chronik im Zusammenhang mit einem Prager Bericht über das Polarlicht vom 10. September 1580 fest, die Himmelserscheinung sei „,von villen glaubwürdigen Leütten gesehen worden, wie dann solliches ein fürnemme Person solches mit aygner handtschrifft bezeugett" ${ }^{75}$

Die zuletzt zitierten Passagen offenbaren nicht nur das Bestreben, die Zuverlässigkeit des Berichteten herauszustellen ${ }^{76}$, sondern machen deutlich, in welchem Maße ,Wunderzeichen" als Katalysatoren kommunikativer Prozesse fungierten. Es dürfte kein Zufall sein, daß der graphische Teil der meisten erhaltenen Flugblätter über Polarlichterscheinungen nicht nur das interessierende Phänomen, sondern auch eine Reihe von meist im Vordergrund befindlichen Betrachterfiguren zeigt, die, wie Körperhaltung und Gestik verraten, über das Gesehene diskutieren. ${ }^{77}$ Daß Polarlichterscheinungen zahlreiche Zuschauer fanden, kann aufgrund der wiederholten Hinweise auf Augenzeugen in publizistischen und chronikalischen Quellen angenommen werden, daß sie darüber hinaus Anlaß zu intensivem mündlichen Austausch boten, findet in Jacob Colers Widmungsvorrede eine Bestätigung. Mit Blick auf die Verunsicherung, die das Polarlicht unter den Mitgliedern seiner Kirchgemeinde offenbar ausgelöst hat, betont er, seine Schrift sei nicht nur als Bußaufruf gedacht, sondern strebe auch eine konsolatorische Wirkung an. All jene verängstigten Gläubigen, die sich mit der Bitte um ein seclsorgerliches Gespräch an ihn gewandt hätten, habe er ,hier-

74 Coler, Notwendige Erinnerung (wie Anm. 58), Biijr-Biijv.

75 Chronik Georg Kölderer (wie Anm. 46), fol. 84r.

${ }^{76}$ Daß3 dies angesichts der bisweilen fragwürdigen Berichte in Flugblätern und Flugschriften und der dadurch bedingten Verunsicherung der Rezipienten frühneuzeillicher Publizistik ciner Notwendigkeit entsprach, stellt Maurer, Georg Kölderer (wie Anm. 45), 49-69, mit Blick auf Georg Kölderer heraus.

77 Dies gilt nicht nur für das vorgängig beschriebene Blatt aus Bartholomäus Käppelers Offizin, sondern auch für einen in Nürnberg veröffentlichten Einblattdruck über das Nordlicht vom 17. Januar 1572 (vgl. Harms/Schilling [Hrsg.|, Wickiana [ wic Anm. 39], Nr. 26) oder die beim Augsburger Drucker Hans Schultes erschienene Darstellung des Nordlichts vom 6. März 1582 (vgl. ebd. Nr. 139). Auch Weher, Wunderzeichen und Winkeldrucker (wic Anm. 38), cnthält eine Reihe von Einblattdrucken zu Himmelserscheinungen, in deren Bildteil Betrachterfiguren integriert sind (vgl. Bildtafeln 7-12 und 16-19). 
mit $[\ldots]$ trösten $[\ldots]$ wollen “. ${ }^{78}$ Prodigien förderten jedoch nicht nur den mündlichen Austausch, sie bildeten auch den Auslöser und zugleich den Kern schriftlicher Kommunikationsnetze. Wenn Georg Kölderer in seiner Chronik schreibt, das Polarlicht vom 10. September 1580 sei „An villen orthen, Im Teütsch, vnnd Ost Lanndt gesehen worden, alls zu Nürmberg, Leibzig, Hamburg, vnnd an annder mehr orthen"79, dann verdankt er dieses Wissen mit hoher Wahrscheinlichkeit publizistischen Quellen ${ }^{80}$. Noch offenkundiger ist der enge Zusammenhang zwischen Publizistik und Historiographie bei Johannes Wick, der systematisch Flugblätter und Flugschriften zu den ihn interessierenden Ereignissen sammelt und sie in sein Manuskript integriert. ${ }^{81}$ Auch Jacob Coler stellt seine Vertrautheit mit zeitgenössischer Publizistik unter Beweis: In der Widmungsvorrede zu seiner Predigt erwähnt er einige ,Wunder', deren Beschreibung er Flugblättern und Flugschriften entnommen hat, wie der Nachsatz ,So ists auch im öffentlichen Druck“ belegt. ${ }^{82}$

78 Coler, Notwendige Erinnerung (wie Anm. 58), Br.

${ }^{79}$ Chronik Georg Kölderer (wie Anm. 46), fol. 80r.

${ }^{80}$ Den kompilatorischen Charakter von Georg Kölderers Chronik hebt auch Maurer, Georg Kölderer (wie Anm. 45), 39-69, hervor. Neben eigenen Beobachtungen und Reflexionen sowie durch mündliche Vermittlung erfahrenen Informationen enthalten seine Aufzeichnungen vor allem Abschriften publizistischer Vorlagen.

81 Nicht nur mit Blick auf Georg Kölderer, sondern auch am Beispicl der handschriftlichen Chronik des Erlurter Kochs Samuel Fritz ließe sich zeigen, daß auch dort, wo keine Druckwerke beigeheftet wurden, publizistische Vorlagen Eingang in chronikalische Aulzeichnungen fanden. Fritz integriert nicht nur Informationen, die er publizistischen Quellen entnommen hat, sondern versieht seine Chronik mit Bebilderungen, deren graphische Vorlagen zu einem nicht unerheblichen Teil auf illustrierten Einblattdrucken zu finden sind; vgl. Ulrich Rosseaux, Die Kipper und Wipper als publizistisches Ereignis (1620-1626). Eine Studic zu den Strukturen öffentlicher Kommunikation im Zeitalter des Dreißigjährigen Krieges. (Schriften zur Wirtschafts- und Sozialgeschichte, 67.) Berlin 2001, 445-447.

82 Coler, Notwendige Frinnerung (wie Anm. 58), Aiiijr. - Dal3 die Autoren frühneuzeitlicher Publizistik meist auch als deren Leser in Firscheinung traten und demzufolge ein dichtes Netz gegenseitiger Verweise zwischen zeitlich und thematisch benachbarten Drucken beschrieben werden kann, stellt auch Rosseaur. Kipper und Wipper (wie Anm. 81), 436-445, heraus. 
IV.

Der Blick auf ausgewählte Quellen zum Polarlicht vom 10. September 1580 dürfte nicht nur das enge Beziehungsgeflecht zwischen publizistischer Überlieferung, chronikalischer Aufzeichnung und protestantischem Predigtdruck deutlich gemacht haben, er belegt zugleich die bemerkenswerte Kongruenz in der Darstellung und Deutung des klärungsbedürftigen Phänomens. In allen hier berücksichtigten Quellen fällt auf, daß der im wesentlichen übereinstimmenden Beschreibung der Himmelserscheinung bemerkenswert viel Raum gewährt wird. Daß Polarlichter sich bis weit ins 18. Jahrhundert hinein einer wissenschaftlichen Klassifikation entzogen und überdies durch ungewöhnliche Variabilität gekennzeichnet waren, ist keine hinreichende Rechtfertigung für die Ausführlichkeit, mit der sie in frühneuzeitlichen Quellen erörtert werden. Offensichtlich weckten sie die Neugier der im 16. Jahrhundert lebenden Menschen, wie nicht nur die Chroniken Georg Kölderers und Johannes Wicks, sondern auch die zeitgenössische Publizistik belegen. Signifikant ist allerdings nicht nur die Ausführlichkeit, mit der die hier interessierende Himmelserscheinung gewürdigt wird, sondern auch und vor allem die sowohl profan- als auch heilsgeschichtliche Deutung, die sie erfährt. Allen Autoren gilt das Polarlicht vom 10. September 1580 als divines Zeichen, das einerseits im Einklang steht mit politisch-militärischen, ökonomischen und sozialen Katastrophenerfahrungen und andererseits auf das drohende göttliche Gericht vorausweist. Zeitkritik verbindet sich mit dem Aufruf zur Buße, die konfessionellen Konflikte erscheinen als Indikatoren für das in Bälde zu erwartende Ende der Welt.

Es stellt sich nun allerdings die Frage, in welchem Umfang die den Quellen zu entnehmenden Wahrnehmungsmuster historisch realisiert wurden. Auch wenn wir aufgrund der bemerkenswert hohen Zahl gedruckter Flugblätter und Flugschriften annehmen können, daß für derartige Drucke eine vergleichsweise große Nachfrage bestand, gilt es zunächst, die Reichweite der vorgängig untersuchten gedruckten Quellen zu klären. Flugblätter und in noch weit größerem Maßße Flugschriften waren in erster Linie einem hinreichend lesefähigen und kaufkräftigen Rezipientenkreis zugänglich; die ärmeren, nicht alphabetisierten Bevölkerungsschichten hatten, wenn überhaupt, nur dank mündlicher Vermittlung die Möglichkeit, sich mit deren Inhalt vertraut zu machen. ${ }^{83}$

83 Vgl. Michael Schilling, Bildpublizistik in der frühen Neuzeit. Aufgaben und 
Dies gilt nun allerdings nicht für die gesprochene protestantische Predigt, die, in die gottesdienstliche Liturgie eingebettet, auch jene nicht lesefähigen Individuen und Gruppen erreichte, denen der Zugang zu gedruckten Schriften verschlossen war. Dafür, daß das Polarlicht vom 10. September $1580 \mathrm{im}$ Gottesdienst erörtert wurde, finden sich in unseren Quellen gleich zwei Belege: Coler betont in seiner Widmungsvorrede, angesichts der Häufung göttlicher Zeichen habe er sich dazu durchgerungen, „euch [den Adressatinnen der Widmungsvorrede, S. S. T.] vnd meinen andern lieben Kirchkindern zu dienst vnd sonder gefallen/ neben vielfeltiger ernster/ aber doch trewer Vermahnung von der Cantzel“ die Predigt in Druck zu geben, ,wiewol“, wie er hervorhebt, ,ich sonsten mit schreiben vnd drucken nicht all zu milde". 84 Auch Georg Kölderer, der in seinem Bericht erwähnt, einer der Augenzeugen, ein Prager Pfarrer, habe seine Beobachtungen ,alls baldt denselben Sontag, offentlich auff der Cantzell gemelldet, vnnd mit seinem gewissen betheurett" ${ }^{\text {" }} 5$, bestätigt, daß die Auseinandersetzung mit unerklärlichen Himmelserscheinungen sich auch und gerade auf den Kirchenraum ausdehnte.

Wir können demnach davon ausgehen, daß nicht nur der Kreis der illiterati, sondern auch weite Teile der nicht alphabetisierten Bevölkerung mit jenen Darstellungs- und Deutungsmustern konfrontiert wurden, die in den zeitgenössischen schriftlichen Quellen begegnen. Inwieweit sie dic damit einhergehenden Vorstellungen im Modus einer affirmativen Perzeption verinnerlichten, kann am ehesten erschlossen werden, wenn man sich die Funktionsweise publizistischer bzw. gottesdienstlicher Kommunikation vergegenwärtigt. Frühneuzeitliche Publizistik folgt den Gesetzen des Marktes. ${ }^{86}$ Wenn deren Produzenten den erwünschten Absatz an Drucken erreichen wollten, mußten sie sich darum bemühen, die Bedürfnisse ihrer Adressaten zu befriedigen, in-

Leistungen des illustrierten Flugblatts in Deutschland bis um 1700. (Studien und Texte zur Sorialgeschichte der Literatur, 29.) Tübingen 1990, 40-53. Eine sorgfailtige Rekonstruktion potentieller und historischer Rezipienten frühneuzeitlicher $\mathrm{Pu}$ blizistik hat kürzlich Rosseatux, Kipper und Wipper (wic Anm. 81), 415-447, veröflentlicht.

${ }^{84}$ Coler, Notwendige Erinnerung (wie Anm. 58), Aiiijv.

85 Chronik Georg Kölderer (wic Anm. 46), fol. 84r.

86 Den Warencharakter von Flugblättern hat bereits Schilling, Bildpublizistik (wic Anm. 83), 11-90, herausgestellt. Vgl. auch Ulrich Rosseaux diesbezügliche Untersuchungen zur Kipper- und Wipperpublizistik (Rosseaux, Kipper und Wipper [wic Anm. 81], 115-268). 
dem sie jene Informationen bereithielten, auf die potentielle Käufer erpicht waren. Die Verfasser von Einblattdrucken und Flugschriften wiederum werden bestrebt gewesen sein, Informationen auf eine Art und Weise zu vermitteln, die deren Glaubwürdigkeit und Relevanz herausstellte. Es ist so gesehen kein Zufall, daß Coler sich als verläßlichen Augenzeugen inszeniert und auf dem Wahrheitsgehalt des Berichteten insistiert. Wenn er sich in der an die Beschreibung des Polarlichts anschließenden Auslegung des Ereignisses immer wieder auf die Bibel beruft, um seine Botschaft zu untermauern, erfährt das Dargelegte eine zusätzliche Beglaubigung. Daß der protestantische Geistliche sich auß3erdem in seiner Funktion als Gott gegenüber zur Rechenschaft verpflichteter Theologe und Seelsorger zu erkennen gibt, verleiht seinen Ausführungen eine fast unantastbare Autorität. Es ist demnach anzunehmen, daß die vom Leser geforderte Rezeptionshaltung, die nicht durch kritische Hinterfragung des in der Flugschrift Dargelegten, sondern vielmehr durch die Bereitschaft, das Gelesene als wahr zu betrachten und sich damit zu identifizieren, gekennzeichnet ist, eine historische Realisierung erfuhr. Der in der Chronik des Johannes Wick bezeugte Umgang mit publizistischen Quellen läßt im übrigen den Schluß $z u, d a ß$ auch gelehrte Leser der Macht des geschriebenen Wortes vertrauten und den Medienerzeugnissen, denen sie ihr Wissen verdankten, Glauben schenkten.

Noch wirkungsmächtiger als das gedruckte dürfte das im Rahmen eines Gottesdienstes gesprochene Wort gewesen sein. ${ }^{87}$ Zum einen wurde das Predigtamt durch einen Geistlichen versehen, der, gerade im protestantischen Verständnis, als von Gott eingesetzter Diener des Wortes fungierte und damit von höchster Stelle autorisiert erschien. Das

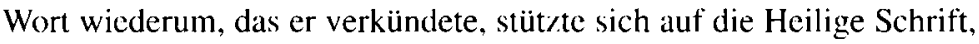
deren Legitimationskraft für die meisten Menschen in der Frühen Neuzeit außer Zweifel stand. Wenn man außerdem in Betracht zieht, welche persuasiven Möglichkeiten die mündliche Rede einem rhetorisch gebildeten Prediger bietet, kann kaum ein Zweifel daran bestehen, daß eine Predigt jeweils intendierte Wirkungen mit hoher Zuverlässigkeit erreichte. Dic sowohl in chronikalischer wie auch publizistischer Überlieferung sich manifestierenden Darstellungs- und Deutungsmuster des

87 Zur Autorität des Predigers vgl. die allerdings primär mit Blick auf die katholische Predigt entwickelten Überlegungen von Franz $M$. Eybl. Abraham a Sancta Clara. Vom Prediger zum Schriftsteller. (Frïhe Neuzeit, 6.) Tübingen 1992, $118-$ 124. 
Polarlichts vom 10. September 1580 dürften demnach in den Wahrnehmungsmodi ihrer zeitgenössischen Rezipienten eine Entsprechung gefunden haben, wofür nicht nur die Dichte und Homogenität der Quellen als geschichtlicher ,Repräsentationen', sondern auch die ,Praktiken", die den zeitgenössischen Umgang mit diesen ,Repräsentationen' bestimmten, sprechen. Die vorgängig untersuchten Quellen haben, dafür bieten sowohl erhaltene Rezeptionsspuren als auch die damit verbundenen Kommunikationssituationen Anhaltspunkte, mit großer Wahrscheinlichkeit eine Perzeption erfahren, die mit den darin zum Ausdruck gelangenden Vorstellungsmodi in Einklang stand. Zwar ließen sich mit Blick auf die meist lateinischen und damit nur einem begrenzten Kreis von Gelehrten zugänglichen Schriften zu astronomischen und geophysikalischen Phänomenen konkurrierende Wahrnehmungs- und Deutungsmuster beschreiben ${ }^{88}$, im Bewußtsein der meisten, wohl nicht nur protestantischen, Betrachter dominierte jedoch die Überzeugung, Himmelserscheinungen seien als göttliche Zeichen auf ihre Botschaft hin zu befragen und für die eigene Lebenswelt nutzbar zu machen.

Eine erschöpfende Analyse und Kontextualisierung publizistischer und chronikalischer Überlieferung zum Polarlicht vom 10. September 1580 vorzulegen, war nicht dic Absicht dieses Beitrags. Daßs sich meine Beobachtungen auf eine vergleichsweise schmale und überdies ausschließlich protestantischen Autoren zu verdankende Quellenbasis stützen, daß einige Aspekte ausgeblendet blieben, die im Zusammenhang mit der zu Beginn meiner Ausführungen entwickelten Fragestellung Aufmerksamkeit verdienen, soll nicht bestritten werden. Welche Rolle unbewußte Stimuli als Katalysatoren von Wahrnehmung spielen, blieb ebenso ungeklärt wic die mit Blick auf die Predigtpraxis nicht unwichtige Frage nach der Signifikanz von Ritualen als Gegenstand menschlicher Wahrnehmung. Unerwähnt geblieben sind des weiteren die perzeptionssteuernden Kontinuitäts- bzw. Traditionsbildungen, die auch im Falle der hier interessicrenden Polarlichterscheinung dazu dicnen, ein cinzclnes Ereignis in einen größeren Zusammenhang einzuordnen; nicht reflektiert wurden die Unterschiede, die zwischen der Wahrnehmung textueller und der Wahrnehmung visueller Zeichen bestehen,

88 Allerdings hat bercits Senn. Johann Jakob Wick (wie Anm. 50), 72, darauf hingewiesen. dad3 religiöse Deutungsmuster auch in wissenschafulich ausgerichtete Werke Kingang finden konnten. Dies gilt, wie Cittwald. Prodigium (wie Anm. 42). 255 f.. gezeigt hat, beispielsweise für Conrad Gessners ..Historia et Interpretatio Prodigii". 
obwohl die meisten der benutzten Quellen durch eine Kombination von Schrift und Bild charakterisiert sind. Dennoch lassen sich aus den bisherigen Darlegungen eine Reihe von Überlegungen und - daraus resultierend - Prinzipien formulieren, die für die Erforschung historischer Perzeptionen leitend sein sollten:

Ein erstes Postulat lautet, daß Texte und Bilder nicht nur historische Wahrnehmungen repräsentieren, sondern daß sie auch historische Wahrnehmungen generieren. Wahrnehmungsgeschichte zu betreiben, bedeutet deshalb, eine Quelle nicht nur als Dokument eines spezifischen Aneignungsmodus historischer Ereignisse zu lesen, sondern zugleich die vielfältigen Praktiken, die sich mit deren zeitgenössischer Rezeption verbinden, in den Blick zu nehmen. Da Quellen durch semantische Mehrschichtigkeit gekennzeichnet sind, bedarf es der Suche nach Rezeptionsspuren, um deren geschichtliche Signifikanz zu erfassen. Erst die Rekonstruktion historischer Perzeptionsmodi und die Verortung einer Quelle in ihrem jeweiligen Kommunikationszusammenhang erlaubt es, deren Bedeutung und deren Funktion(en) adäquat zu beschreiben. Daß es nicht genügt, einzelne Quellen isoliert zu betrachten, haben meine Ausführungen zu ausgewählten Reaktionen auf das Polarlicht vom 10. September 1580 deutlich zu machen versucht. Gerade mit Blick auf Predigten und publizistische Quellen gilt es, die Kommunikationszusammenhänge zu rekonstruieren, in denen deren Rezeption eingebunden ist. So stützt der Umstand, daß frühneuzeitliche Publizistik verkauft wurde und der kommerzielle Erfolg nur gewährleistet war, wenn sie die Erwartungen und Bedürfnisse der Käufer zu befriedigen vermochte, die Annahme, daß zwischen den in illustrierten Einblattdrucken und Flugschriften bereitgestellten Sinnstiftungsangeboten und dem Wissens- und Wahrnehmungshorizont der Rezipienten eine zumindest partielle Kongruenz bestand, und das Wissen um den autoritativen Charakter gottesdienstlicher Rede erleichtert es, die Wirkung der in Predigten zum Ausdruck kommenden theologischen Interpretation astronomischer Phänomene zu plausibilisieren.

Die hier angesprochene semantische Mehrschichtigkeit historischer Überlieferung führt zu einem zweiten Postulat: Die Analyse schriftlicher und bildlicher Quellen hat im Bewußtsein ihres medialen Charakters zu erfolgen. Quellen liefern keine eineindeutigen Aussagen, sie unterliegen gattungsbedingten Stilisierungen, verbergen hinter offenkundigen Intentionen verborgene Botschaften, weisen Leerstellen auf, welche die Deutungskompetenz des Wissenschaftlers herausfordern. 
Nun ist die Interpretationsbedürftigkeit geschichtlicher Überlieferung zwar ein generelles Problem historischer Forschung; für die Kulturgeschichte, die traditionell von einem weiten Quellenbegriff ausgeht, stellt sie nachgerade die zentrale wissenschaftliche Herausforderung dar. Wer, wie dies zahlreiche kulturhistorisch ausgerichtete Studien tun, auf künstlerische Artefakte, beispielsweise Romane oder Dramen, Gemälde und Skulpturen oder musikalische Werke, zurückgreift und publizistische, philosophische, wissenschaftliche Quellen heranzieht, um geschichtlich sich ausformende menschliche Vorstellungswelten zu untersuchen, sieht sich in besonderem Maße vor die Aufgabe gestellt, die mit diesen Quellen in Zusammenhang stehenden Gestaltungs- und Wahrnehmungskonventionen zu bedenken.

Der Hinweis auf künstlerische Artefakte sowie publizistische, philosophische und wissenschaftliche Überlieferung als mögliche Quellen wahrnehmungsgeschichtlicher Forschung impliziert ein drittes Postulat: Eine Beschränkung auf die Erforschung kollektiver, Vorstellungsmuster ${ }^{*}$ unterer sozialer Schichten, wie sie für nicht wenige Vertreter der histoire des mentalités kennzeichnend ist, erweist sich gerade für wahrnehmungsgeschichtliche Fragestellungen als unbefriedigend. Wer sich auf Quellen stützt, um historische Wahrnehmungen zu rekonstruieren, wird vor allem mit Dokumenten zu tun haben, die sich Individuen aus den politisch und ökonomisch einflußreichen oder zumindest den gelehrten Schichten verdanken. Die Erforschung der Perzeptionsmodi nur rudimentär gebildeter Angehöriger nichtprivilegierter Bevölkerungsgruppen ist zwar nicht unmöglich, wie Carlo Ginzburgs berühmt gewordene Studie über die Vorstellungswelt eines norditalienischen Müllers auf eindrucksvolle Weise belegt ${ }^{89}$, aber sie kann, auch das macht Ginzburgs Buch deutlich, nicht losgelöst von den gelehrten Wissensbeständen des untersuchten Zeitraums erfolgen. ,Elite' und ,Volk ' bilden hinsichtlich ihrer kulturellen Erfahrungen und Praktiken keine völlig autonomen Entitäten, zwischen den verschiedenen sozialen Gruppen besteht vielmehr ein komplexer und dynamischer Austausch von Wissen und Wahrnehmungen. Für Wahrnehmungshistoriker bedeutet dies, daß sie gelehrte und populäre Perzeptionsmuster sowohl im Kontext ihrer jeweiligen sozialen und kulturellen Konventionen als auch in ihrer gegenseitigen Vernetzung zu ergründen haben.

89 Carlo Ginzhurg, Der Käse und die Würmer. Die Welt cines Müllers um 1600 . Aus d. Ital. v. Karl F. Hauber. Frankfurt am Main 1979 |ital. 1976|. 
Daran anschließend läßt sich schließlich ein viertes Postulat formulieren: Eine Konzentration ausschließlich auf kollektive Wahrnehmungen, wie dies einige Historiker der Annales propagiert haben, oder aber ausschließlich auf individuelle Wahrnehmungen, wie sie die histoire intellectuelle praktizierte, verhindert eine umfassende Einsicht in historische Perzeptionsmuster. Der Blick des Wahrnehmungshistorikers hat sich sowohl auf individuelle als auch kollektive Wahrnehmungen zu richten. Dabei scheint es sinnvoll, zunächst von einer sorgfältigen Rekonstruktion individueller Wahrnehmungen auszugehen. Jede individuelle Wahrnehmung nun enthält, wie Winfried Schulze es ausgedrückt hat, ein ,schwer quantifizierbares Moment an Vergesellschaftung"90, und verweist damit bereits auf kollektive Wissensbestände, Wahrnehmungs- und Deutungsmuster. Erst durch die Verknüpfung einer größeren Zahl individueller Wahrnehmungen lassen sich allerdings die in einem spezifischen Moment und in einem spezifischen kulturellen Raum dominierenden Perzeptionsmodi eruieren und in Verbindung setzen zu politischen, ökonomischen und sozialen Entwicklungen. Die auch und gerade Kulturhistorikern keinesfalls fremde Vorstellung, das Ergebnis derartiger Bemühungen sei die Einsicht in jene fundamentalen Ideen, welche einer Epoche ihre spezifische Eigenart und ihre Homogenität verleihen würden, dürfte sich allerdings als Illusion entpuppen. Ungeachtet der Tatsache, daß, wie das vorgängig dargelegte Fallbeispiel gezeigt hat, zeitgenössische Perzeptionen eines Ereignisses bisweilen eine bemerkenswerte Kongruenz aufweisen können, gilt es deshalb, für eine wahrnehmungsgeschichtliche Forschung zu plädieren, die nicht nur die dominierenden Wahrnehmungs- und Deutungsmuster einer Zeit erhellt, sondern die auch gegenläufige Positionen und Widersprüche in den Blick nimmt und sich der gerade für Historiker besonders irritierenden und faszinierenden Erfahrung der Gleichzeitigkeit des Ungleichzeitigen stellt.

${ }^{90}$ Winfried Schulze, Mikrohistorie versus Makrohistorie? Anmerkungen zu einem aktuellen Thema, in: Christian Meier/Jörn Rüsen (Hrsg.), Historische Methode. (Theoric der Geschichte. Beitrïge zur Historik, 5.) München 1988, 319-341, hier 337. 


\section{Zusammenfassung}

Angesichts der Tatsache, daß die Kulturgeschichte seit jeher ihre $\mathbf{Z u}$ ständigkeit für jene mentalen Dispositionen, die historisches Handeln determinieren, postuliert, stellt die Klärung der Frage, wie etwas derart Geschichtsmächtiges und zugleich Ungreifbares wie menschliche Wahrnehmung als wissenskonstituierende und handlungsleitende Operation auf wissenschaftlich plausible Weise rekonstruiert werden kann, eine vordringliche Aufgabe kulturhistorischer Reflexion dar. Ausgehend von in Auseinandersetzung mit Positionen der französischen Mentalitätengeschichte entwickelten Überlegungen des Historikers Roger Chartier erörtert der Beitrag einen kommunikationsgeschichtlichen Lösungsansatz und erprobt ihn an einem exemplarischen Fall: Das Beispiel eines chronikalisch und publizistisch vielfältig bezeugten Polarlichts, das im Jahre 1580 in fast ganz. Mitteleuropa zu sehen war, macht deutlich, daß die Rekonstruktion historischer Wahrnehmungsmodi, verstanden als aktive und bewußte Akte der Wissenskonstitution und Wirklichkeitsdeutung, nicht nur von den - in den Worten Chartiers - ,Repräsentationen', d.h. den spezifische Perzeptionsweisen dokumentierenden Quellen, ausgehen darf, sondern auch die die Genese und den zeitgenössischen Umgang mit einer Quelle bestimmenden kulturellen Handlungsweisen erforschen muß Nur eine Analyse, so die These, welche die sozialen, institutionellen und kulturellen Determinationen und die damit verbundenen kommunikativen Praktiken gleichermaßen in den Blick nimmt, eröffnet dem Historiker die Möglichkeit, individuelle und kollektive Wahrnehmungsmuster methodisch überzeugend zu rekonstruicren und deren jeweilige geschichtliche Relevan\%. zu bestimmen. 


\section{Die Berliner Universität in der NS-Zeit}

BAND I

Strukturen und Personen

Herausgegeben von Christoph Jahr unter Mitarbeit von Rebecca Schaarschmidt

BAND ॥

\section{Fachbereiche und Fakultäten}

Herausgegeben von Rüdiger vom Bruch unter Mitarbeit von Rebecca Schaarschmidt

Band I:

2005. 257 Seiten mit 3 Abbildungen. Kart. €23,- / sFr 36,80. ISBN 3-515-08657-9

Band II:

2005. 308 Seiten mit 10 Abbildungen. Kart. €23,- / sFr 36,80. ISBN 3-515-08658-7

Das Interesse an der Geschichte der Wissenschaftsdisziplinen und Universitäten in der NS-Zeit ist seit einigen Jahren enorm gewachsen. Die Humboldt-Universität zu Berlin hat sich der Aufarbeitung ihrer Rolle in der NS-Zeit in einer Ringvorlesung angenommen, die diesem Sammelband zugrunde liegt. Zahlreiche der Beiträge bieten dabei erste Forschungsergebnisse zu ihren Themen überhaupt. In den 14 Aufsätzen des ersten Bandes geht es um das Verhältnis von institutionellen sowie personellen Brüchen und Kontinuitäten nach 1933.

Die Aufsätze des zweiten Bandes geben Einblick in Fakultäten und Institute der Berliner Universität.

Ebenfalls lieferbar: Rüdiger vom Bruch

\section{Bürgerlichkeit, Staat und Kultur im Deutschen Kaiserreich}

Herausgegeben von Hans-Christoph Liess

2005. 394 Seiten. Kart. $€ 68,-$ / SFr 108,80. ISBN 3-515-08656-0

\section{„... immer im Forschen bleiben! “}

Rüdiger vom Bruch zum 6o. Geburtstag

Herausgegeben von Marc Schalenberg und Peter Th. Walther 2005. 433 Seiten. Geb. $€ 68,-/$ SFr 108,80. ISBN 3-515-08607-2

\section{Franz Steiner Verlag}

Neuere Geschichte 